\title{
Model-driven development of asynchronous message-driven architectures with AsyncAPI
}

\author{
Abel Gómez ${ }^{1}\left(\mathbb{D} \cdot\right.$ Markel Iglesias-Urkia $^{2} \cdot$ Lorea Belategi $^{2} \cdot$ Xabier Mendialdua $^{2} \cdot$ Jordi Cabot $^{3}$
}

Received: 30 October 2020 / Revised: 15 October 2021 / Accepted: 20 October 2021 / Published online: 2 December 2021

(c) The Author(s) 2021

\begin{abstract}
In the Internet-of-Things (IoT) vision, everyday objects evolve into cyber-physical systems. The massive use and deployment of these systems has given place to the Industry 4.0 or Industrial IoT (IIoT). Due to its scalability requirements, IIoT architectures are typically distributed and asynchronous. In this scenario, one of the most widely used paradigms is publish/subscribe, where messages are sent and received based on a set of categories or topics. However, these architectures face interoperability challenges. Consistency in message categories and structure is the key to avoid potential losses of information. Ensuring this consistency requires complex data processing logic both on the publisher and the subscriber sides. In this paper, we present our proposal relying on AsyncAPI to automate the design and implementation of these asynchronous architectures using model-driven techniques for the generation of (part of) message-driven infrastructures. Our proposal offers two different ways of designing the architectures: either graphically, by modeling and annotating the messages that are sent among the different IoT devices, or textually, by implementing an editor compliant with the AsyncAPI specification. We have evaluated our proposal by conducting a set of experiments with 25 subjects with different expertise and background. The experiments show that one-third of the subjects were able to design and implement a working architecture in less than an hour without previous knowledge of our proposal, and an additional one-third estimated that they would only need less than two hours in total.
\end{abstract}

Keywords Publish/subscribe · Cyber-physical systems (CPS) · Message-driven architectures · Asynchronous communication · AsyncAPI · Industrial Internet of Things (IIoT)

Communicated by Federico Ciccozzi, Nicolas Ferry, Arnor Solberg, and Manuel Wimmer.

Abel Gómez

agomezlla@uoc.edu

Markel Iglesias-Urkia

miglesias@ikerlan.es

Lorea Belategi

lbelategi@ikerlan.es

Xabier Mendialdua

xmendialdua@ikerlan.es

Jordi Cabot

jordi.cabot@icrea.cat

1 Internet Interdisciplinary Institute (IN3), Universitat Oberta de Catalunya (UOC), Barcelona, Spain

2 Ikerlan Technology Research Centre, Basque Research and Technology Alliance (BRTA), Arrasate-Mondragón, Spain

3 ICREA - Internet Interdisciplinary Institute (IN3), Universitat Oberta de Catalunya (UOC), Barcelona, Spain

\section{Introduction}

The emergence of the Internet of Things (IoT) [18] has dramatically changed how physical objects are conceived in our society and industry. In the new era of the IoT, every object becomes a complex cyber-physical system (CPS) [27], where both the physical characteristics and the software that manages them are highly intertwined. Nowadays, many everyday objects are in fact CPSs, which increasingly use sensors and interfaces (APIs) to interact and exchange data with the cloud [45].

The ideas behind the IoT have been especially embraced by industry in the Industrial IoT (IIoT) or Industry 4.0 [28]. It is in this Industry 4.0 scenario where CPSs become especially relevant, mainly in control and monitoring tasks [31].

IIoT has largely contributed to the growing interest in message-driven architectures [33], best used for asynchronous communication. Indeed, in order to achieve higher degrees of scalability, CPSs are typically deployed on 
message-driven asynchronous architectures that improve the overall behavior and reliability of systems. One of the most popular paradigms today is publish/subscribe [1]-followed by, for example, the Message Queuing Telemetry Transport (MQTT) protocol-where messages that are sent to and from a CPS are not directed to a certain recipient, but are proactively published and consumed by the agents involved according to certain criteria or categories. However, although these distributed architectures are especially scalable and tolerant to changes, they are not problem-free: since communication is done between equals, there must be an agreement between all parties on what are the expected message categories, as well as on their internal format and structure.

This is a key challenge we face at Ikerlan. As a technology center, Ikerlan currently coordinates different projects developing solutions to monitor, control and supervise systems of remote IoT devices in manufacturing plants, consumer goods, warehouses or smart buildings. Such solutions must support environments where a large number of devices send and consume data (e.g., sensor information, batch processed data, etc.). Following the current trend, the solutions developed are based on message-driven architectures following the publish/subscribe paradigm.

Based on our experience in working on a large number of IoT projects of different sizes and domains, we propose a model-driven solution to design and develop these architectures efficiently. Our proposal relies on the AsyncAPI Specification [5] to formalize and (semi)automate the design and implementation of these architectures. Previously [21], we presented both AsyncAPI-based concrete and abstract syntaxes and code generation templates to generate a Java-based internal DSL to support message-driven architectures. In this paper, we generalize and extend our proposal, and we provide further details. A key extension not presented before is the graphical concrete syntax based on data models together with a set of model transformation to kick start the modeling and development of messagedriven architectures. The transformations also guarantee the interoperability between the different syntaxes to ensure that developers can, for instance, start with the graphical one and then refine the result with the textual representation. These extensions have been implemented and integrated in our toolkit.

Furthermore, this paper completes our previous work by reporting on the evaluation of our toolkit. The evaluation we present here has been carried out with a group of 25 subjects - both end-users and developers-with different levels of knowledge in the required concepts. Specifically, we asked them to implement a small example architecture of an automated warehouse. The goal of this evaluation was to answer the following research questions: (i)how do users think our toolkit helps them in the definition and development of an asynchronous message-driven architectures? (ii)is previous knowledge required to use our toolkit? (iii)which are the weaknesses and strengths of our toolkit? and, (iv)does the graphical syntax provide real benefits?

To answer these questions, the experiment was conducted in two phases: first, we evaluated the usefulness of the initial tool and got the feedback of the participants using a questionnaire, and second, after evaluating the initial results, we selected the 11 subjects with previous knowledge on modeling and asked them to use the graphical syntax to implement the same exercise to evaluate the extensions done. The complete prototype is available as an open-source toolkit freely available online ${ }^{1}$.

The rest of this paper is structured as follows: Section 2 motivates this work by introducing a small use case that will be used as a running example throughout the rest of the paper, while Sect. 3 presents AsyncAPI, which serves as the background for this work. Section 4 presents our proposed workflow for designing and developing messagedriven architectures as well as the overall picture of our proposed tool, and Sect. 5 presents in detail our tool, describing all the main artifacts and components involved. Section 6 presents how we validated our proposal, first discussing on our initial experiences and internal evaluation and second by presenting our experiments with external subjects. Section 7 presents the related work. We finish the paper with Sect. 8, where we present our conclusions and summarize our roadmap.

\section{Motivation}

Monitoring and control needs, as well as security and reliability requirements, make possible — and even desirable - to reuse a generic reference architecture in IIoT environments. Architectures in these environments are typically messagebased, thus allowing a low coupling among the elements in the architecture. One common message-based paradigm is publish/subscribe, where messages are not directly sent to the recipients who will consume them, but are published under a certain category called topic. Only the devices subscribed to a certain topic will receive the messages published under it.

A common use case of these message-based architectures is IoT devices publishing monitoring and status data. Such data, whose volume can be very high, may be consumed by a cloud application that filters and processes it. On the other hand, control messages may be sent to reconfigure the IoT devices through a Frontend when needed. The central element of this architecture would be the message broker: the

\footnotetext{
${ }^{1}$ https://hdl.handle.net/20.500.12004/1/A/ASYNCAPI/001.
} 


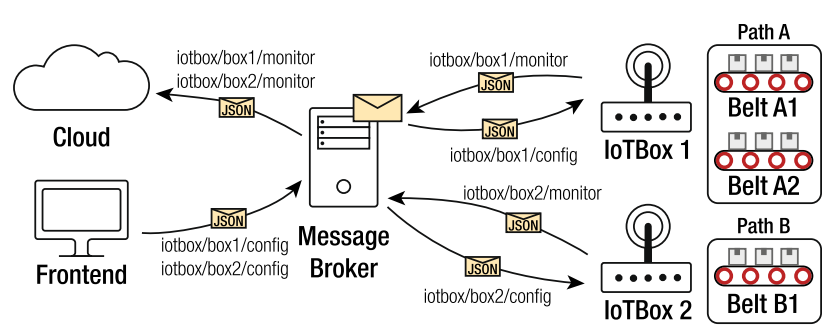

Fig. 1 Example of a message-based architecture in IoT

element in charge of managing publications, subscriptions, and the flow of messages between the elements of the network.

Example In order to illustrate the kind of architectures explained above, we will use a simplified use case of an automated warehouse from an industrial partner as shown in Fig. 1. The message broker is shown in the center of the figure, and as aforementioned, it is in charge of managing the different publications and subscriptions. The warehouse has different paths, each one containing a varying number of conveyor belts, as depicted on the right-hand side of the figure. IoTBoxes are IoT devices which are distributed throughout the factory and are capable of monitoring and controlling different paths. In the example, IoTBox 1 controls and monitors Path $A$ (and thus belts $A 1$ and $A 2$ ), while IoTBox 2 controls and monitors Path $B$ (and as a consequence, belt $B 1$ ). The IoTBoxes periodically collect data from the belts in the path - e.g., speed values. This datum is sent to be further processed in the cloud. To do this, IoTBox 1 publishes its monitoring information in topic iotbox/box1/monitor, while IoTBox 2 does the same on topic iotbox/box $2 /$ monitor. To receive the monitoring data, the cloud is subscribed to topic iotbox/+/ monitor - the + symbol acts as a wildcard—and as a consequence, the cloud receives the monitoring data of both IoTBoxes under topics iotbox/box1/monitor and iotbox/box $2 / m o n i t o r$.

IoTBoxes may also receive configuration commands-for example, to change the monitoring frequency. These configuration commands can be issued remotely via the frontend. For example, the frontend may publish the desired monitoring frequency for Path $A$-which is controlled by IoTBox 1by publishing a message with the correct format under topic iotbox/box1/config. Since IoTBox 1 is subscribed to this topic, it will receive the configuration command and will reconfigure itself as requested. The same applies to IoTBox 2 and Path B, but under topic iotbox/box2/ config in this case.

As it can be guessed by examining the example above, one of the major challenges that these architectures pose is consistency [22]: the format of the messages exchanged and the topics under which they are published and subscribed

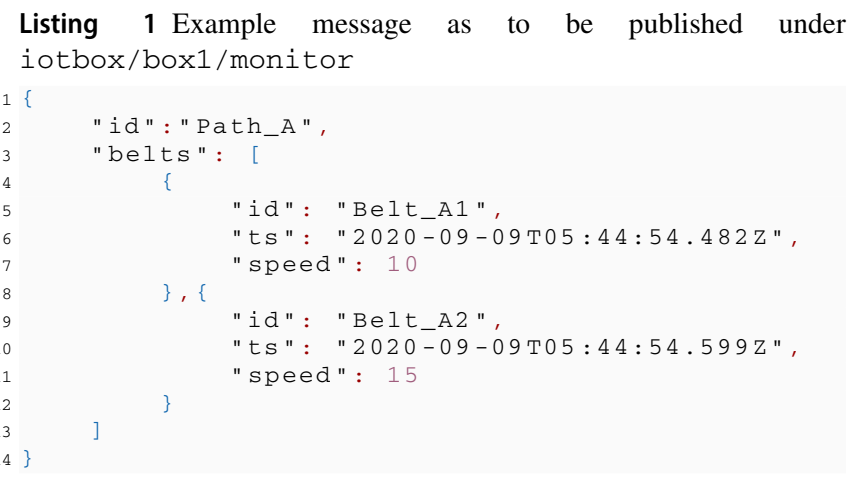

must be kept consistent throughout the life cycle of the system. Failure to comply with this could result in a system malfunction: if any of the agents introduces (even minimal) changes in the definition of communications, and these changes are not propagated to all the agents involved, interoperability problems will inevitably occur. Communication can cease to be effective for two reasons: (i) because there is a divergence in the topics under which messages are published, thus resulting in agents not receiving messages they are interested in; or (ii) because there is a divergence in the format of the messages of a certain topic, and therefore, these cannot be understood by the subscribers receiving them.

Example Listing 1 shows a well-formed message as it is published by IoTBox 1 under topic iotbox/box1 /monitor. The message contains a JSON object with two fields: $i d$ and belts. id identifies the path, and belts is an array of objects, each one containing three fields: a belt $i d$, the timestamp ( $t s$ ) when the speed was observed on the belt, and the speed of the belt in $\mathrm{m} / \mathrm{s}$. An example of divergence in the topics is the case when the cloud subscribes to the wrong topic by mistake (e.g., to iotbox/box1 / moni toring instead of iotbox/box1/monitor). In such a case, the monitoring data of IoTBox 1 will not be delivered to the cloud ever. An example of divergence in the format can happen if the developers coding the cloud application do not pay attention to the message format. For example, if the speed is treated as a float, precision errors may occur or even the parsing may fail; or if the timestamp is treated as a long with the epoch time (instead of as a formatted string) even a runtime error may happen.

\section{AsyncAPI: Towards a standard language for describing message-based architectures}

Aforementioned consistency issues are not unique to messagebased architectures where communication occurs asyn- 
chronously. In fact, other architectures also manifest them, as it is the case of resource-oriented architectures where communication occurs synchronously. However, in these cases, the industry has already proposed standardized solutions to support the development of such architectures. An example is OpenAPI and its complete ecosystem. The OpenAPI Specification [41] is a description format for APIs based on the REST [19] paradigm that allows, among other things, to specify the operations offered by the API, the type and parameters of each operation, the authentication methods, etc.

For message-based architectures, and taking inspiration from OpenAPI, the AsyncAPI Specification [5] proposal has recently emerged as a promising alternative. AsyncAPI descriptions are expected to be both human and machine readable. To achieve this goal, files defining a message-driven API are represented as JSON objects and conform to the JSON standards ${ }^{2}$. Such files allow describing, among other things, the message brokers of an architecture, the topics of interest, or the different formats of the messages associated with each of the topics. Next, we introduce the most preeminent concepts-JSON objects in the AsyncAPI Specification - of the AsyncAPI proposal for future reference ${ }^{3}$ :

The AsyncAPI object is the root document object of an API definition. It combines resource listing and API declaration together into one document. Its main fields are: asyncapi, to specify the AsyncAPI Specification version being used; info, an Info object; servers, a Servers object; channels, a Channels object; and components, a Components object.

The Info object provides the API metadata, such as its title, version, description, termsOfService, contact, and license.

The Servers object is a map of Server objects.

A Server object typically represents a message broker (or a similar computer program). This object is used to capture details such as URLs, protocols and security configuration of such brokers. Variable substitution is also supported. The object contains, among other fields, a url to the target host, its protocol (e.g., http, mqtt, stomp, $k a f k a$, etc.), the protocolVersion, a description, or a map of variables.

The Channels object is a map holding relative path names and individual Channel Item objects. Channel path names are relative to servers. Channels are also known as topics, routing keys, event types or paths

2 YAML, being a superset of JSON, can be used as well to represent an AsyncAPI Specification file too.

3 An interested reader may find Fig. 4 useful, where we propose a metamodel for the AsyncAPI Specification based on the concepts presented here. depending on the protocol or technology used.

A Channel Item object describes the operations available on a single channel (i.e., topic). Typical fields are: description, to describe the channel; subscribe, an Operation object; publish, an Operation object too; or parameters, a map of the parameters included in the channel name.

An Operation object describes a publish or a subscribe operation. This provides a place to document how and why messages are sent and received. Most common fields are: operationId, a unique string used to identify the operation; summary, a short summary of what the operation is about; description, a verbose explanation of the operation; and message, a Message object with the definition of the message that will be published or received on this channel.

A Message object describes a message received on a given channel and operation. For a message, the following fields can be specified among other: name, a machinefriendly name for the message; title, a human-friendly title for the message; summary; description; or payload, which can be of any type but defaults to Schema object.

A Schema object allows the definition of input and output data types. These types can be objects, but also primitives and arrays. This object is a superset of the JSON Schema Specification Draft $7^{4}$. Typical fields of an Schema object are: title; type (any of boolean, integer, number, string, object, array or null); enum, to limit possible values from a list of literals; properties, to specify the fields of objects; maxItems and minItems, to specify the cardinality of arrays; or items, to specify the schema of the array elements.

A Reference object is a simple object which allows referencing other components in the specification, internally and externally. It only contains the $\$$ ref field, which is a URI.

The Components object holds a set of reusable objects for different aspects of the AsyncAPI definition. Elements defined within the Components object can be referenced by using a Reference object. Reusable objects are mapped by name in their corresponding field. Some examples are: schemas, for Schema definitions; messages, for Message definitions; parameters, for Parameters; or operationTraits and messageTraits, which are traits that can be applied to operations and messages, respectively, and are defined similarly to Operations and Messages.

\footnotetext{
${ }^{4}$ https://json-schema.org/specification-links.html\#draft-7
} 
Listing 2: AsyncAPI specification for an IoTbox

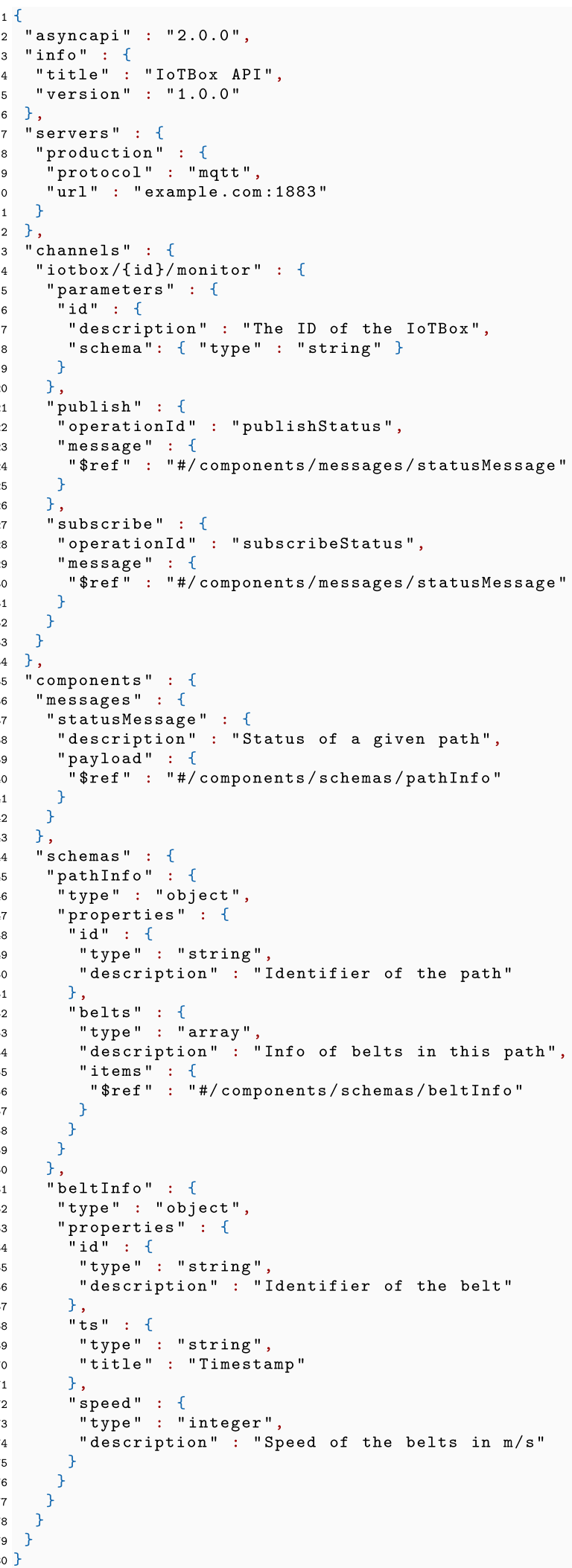

Example Listing 2 shows, in a simplified way, how part of our running example is specified using AsyncAPI. To keep the example manageable, we have specified only the monitoring part (thus excluding the configuration topics-e.g., iotbox/box1/config-and associated messages). As it can be seen, in line 2, we specify that the definition adheres to the AsyncAPI Specification version 2.0.0, while in lines 3-6, we specify the information of our API. As lines 7-12 specify, our infrastructure has a single server, called production - whose host name is example.com-with an MQTT broker listening on port 1883. The rest of the AsyncAPI object specifies the topics exposed by our API, and the format of the messages that can be interchanged. This is done via the channels property (lines 13-34), which in turn, references some reusable artifacts that have been defined within the components property (lines 3579). Line 14 specifies the name of the only channel-i.e., topic_of our infrastructure: iotbox/ $\{i d\} / m o n i t o r$. As it can be guessed, iotbox/ $\{i d\} / m o n i t o r$ is a parameterized name, in which the $\{i d\}$ substring is substituted by the actual IoTBox name when publishing a message (thus publishing either under the iotbox/box $1 /$ monitor or iotbox/box 2/monitor topics). Lines 16-19 specify the actual information of the parameter: in line 16, we specify its name; in line 17, we provide a description; and in line 18, we specify its type. The publish and subscribe operations are specified in lines 21-26 and 27-32, respectively. As both operations publish and receive the same kind of messages, they reference the reusable definition named statusMessage, which is defined in the messages property of the components object (lines 36-43). The payload of a statusMessage is a pathInfo object. The schema of pathInfo objects is specified in lines 45-60. A pathInfo is a JSON object with two properties: $i d$, a string value; and belts, an array of beltInfo objects. As specified in lines 61-77, a beltInfo is an object with three properties: an id; a timestamp (whose name is $t s$ ); and a numeric value with the speed of the belt. As it can be observed, the example message shown in Sect. 2-i.e., Listing 1 -contains a pathInfo object conforming to the specification in lines 45-77.

As it can be observed in the example, the AsyncAPI Specification allows defining all the relevant information needed to design and execute a message-driven API. However, as of writing this manuscript, AsyncAPI is only an emerging proposal and its ecosystem is still flourishing. Some documentation and code generators exist—with different maturity levels ${ }^{5}$ - but still no integrated development workflow to design and implement message-driven architectures has been proposed since transitions between different development tasks must be manually done.

5 See https://shapeup.asyncapi.io to follow the development progress of the different tools under the umbrella of the AsyncAPI Initiative. 


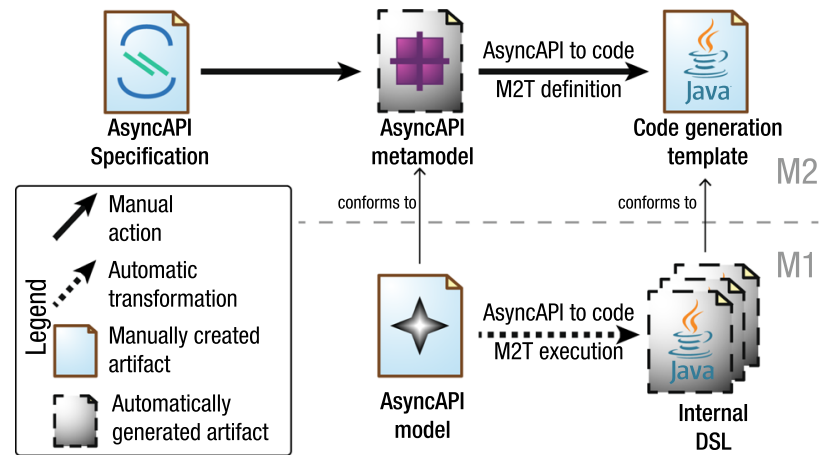

Fig. 2 Generic model-driven process where AsyncAPI serves as the single source of truth

\section{Model-driven development of asynchronous message-driven architectures}

One of the major flaws of message-driven architectures, as Sect. 2 illustrates, is how easily the knowledge about the infrastructure dilutes among all the elements involved in it. As a consequence, it is very easy to introduce divergences on how messages are sent and consumed by the different actors involved. This major flaw can, however, be solved if we use models as the single source of truth used throughout the design, development and execution of the infrastructure.

As we have seen in Sect. 3, despite its current limitations, AsyncAPI provides the grounds to design a complete conceptual framework. But, in order to make an effective use of AsyncAPI, a proper process and infrastructure supporting not only the design, but also the whole life cycle of a message-driven architecture, is still needed; models are the perfect asset to overcome these current limitations. Based on our experience, using model-driven techniques allow us (i) to keep our approach modular and extensible, alleviating a possible vendor lock-in; (ii) to integrate other IoT standards, different programming languages and frameworks, or future projects with not yet known requirements at Ikerlan; and (iii) to boost our productivity by taking advantage of the plethora of model-driven technologies and solutions available in the market-metamodeling frameworks, code generation engines, model transformation engines, etc.

Figure 2 shows what a typical model-driven workflowwith the main involved artifacts represented as a paper sheet-looks like. On the one hand, artifacts depicted with a solid line represent manually created artifacts, while artifacts depicted with dashed lines are automatically generated using different transformations. On the other hand, artifacts at the M1 layer (the model layer) are defined - or automatically created - each time a new development process is enacted, while artifacts at the M2 layer (the metamodel layer) are defined only once during the development of the toolkit itself.
The figure represents that, based on the AsyncAPI Specification, a corresponding AsyncAPI metamodel can be created for its abstract syntax. Using the concepts of this abstract syntax, a model-to-text (M2T) transformation generating executable code can be defined-AsyncAPI to code. Thus, by executing this M2T transformation, a library managing all basic functionality of a message-driven infrastructurethat will be shared by all the elements participating in it-can be generated from an AsyncAPI model. This library can be implemented in such a way that it exposes an internal domain specific language (DSL) that can be used by the client code ${ }^{6}$ to perform tasks such as message creation, message parsing and processing, publications, subscriptions, etc.

\subsection{The AsyncAPI Toolkit: Implementing a model- based development workflow from the beginning}

While this generic workflow provides the grounds for a minimal working solution, it is impractical since it does not consider the concrete syntax for AsyncAPI definitions (which are JSON-based according to the specification) and assumes designers would take care of manually creating the AsyncAPI models from the JSON-based descriptions.

Instead, Fig. 3 shows how we envision a completely practical solution for an improved workflow, extending the previous building blocks and covering all the way from the concrete syntax to the code generation phase. We have implemented this workflow as an open-source solution called AsyncAPI Toolkit also following model-driven development principles: instead of manually developing an AsyncAPI metamodel, we have taken advantage of the Xtext framework [53] to provide both a concrete and an abstract syntax for AsyncAPI.

Thus, based on the AsyncAPI Specification introduced in Sect. 3, we have developed an AsyncAPI JSON grammar in Xtext-see Sect. 5.1-that validates message-driven API definitions conforming to the AsyncAPI Specification. Likewise, from this grammar, Xtext automatically generates the corresponding AsyncAPI metamodel and all the toolingeditor with content assist, parser, etc.- - to easily create and transform AsyncAPI JSON definitions into AsyncAPI models conforming to the AsyncAPI metamodel - see Sect. 5.2. Given the availability of both a metamodel for AsyncAPI and the specification of an API as a conformant model, the workflow can continue as explained above, i.e., by executing a M2T transformation that generates an internal DSL.

\footnotetext{
${ }^{6}$ We understand as client code the actual applications making use of the messages sent and received, and whose logic cannot be captured in the AsyncAPI definition. An example of client code would be the application running in the cloud, which is in charge of processing the monitoring data sent by the IoTBoxes.
} 


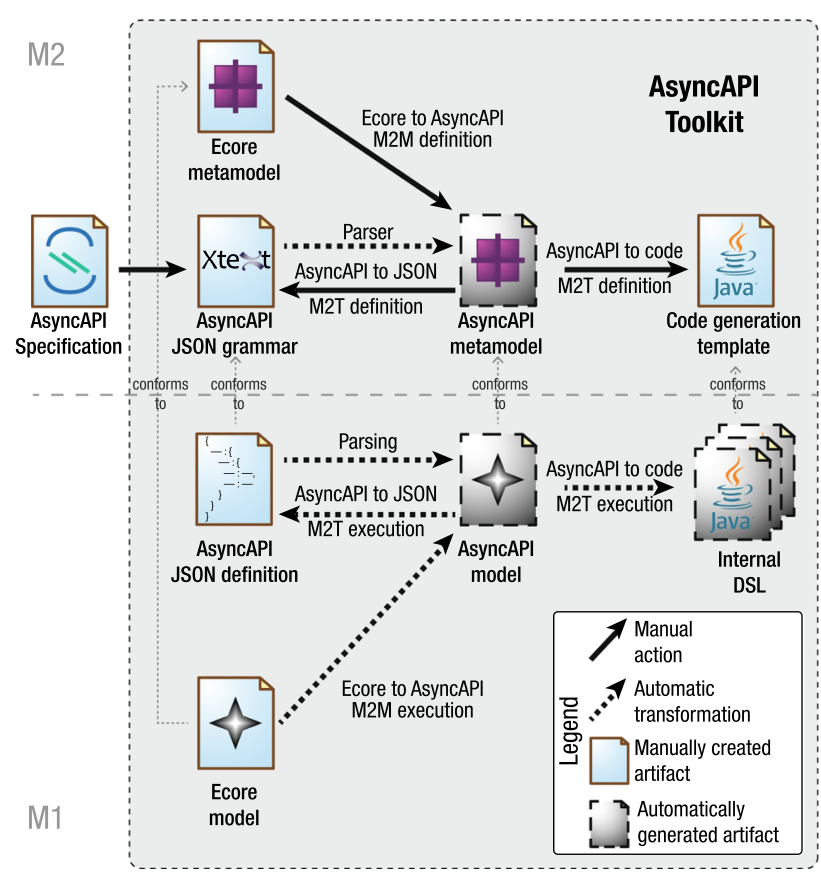

Fig. 3 Actual model-driven development process implemented by AsyncAPI Toolkit with its main involved artifacts

At this moment, the AsyncAPI Toolkit supports the Java language and generates a library that assists developers in the creation, publication and reception of well-formed messages by providing a fluent API - see Sect. 5.5 -as proposed by [20].

\subsection{Bootstrapping a message-driven architecture from data models}

It is noteworthy that, since these architectures are messagebased, data modeling plays a crucial role. For this reason, we have completed the above workflow with an alternative (graphical) concrete syntax focused on the modeling of the messages to be exchanged. Thus, instead of using JSON Schema, messages can be directly specified using Ecore models which conform to the Ecore metamodel provided by the Eclipse Modeling Framework (EMF) [51]. Such Ecore models can be further annotated to capture extra information-channels, servers, etc. - that may be needed to specify a fully working AsyncAPI definition (see Sect. 5.3 for an example). Nevertheless, it must be pointed out that $J S O N$ Schema is more expressive than plain Ecore-e.g., think about the anyOf, oneOf, allOf or not keywords, to name a few - and the same applies to the annotations we have made available in Ecore, which have been limited to the bare minimum. In these cases, data modeling is conceived as a means to bootstrap an AsyncAPI JSON definition that can be later manually refined thus reducing the initial develop- ment time ${ }^{7}$. Besides its limitations, obtaining a JSON-based representation of an Ecore model poses several advantages: (i) allows developers and architects to create a working Asyn$c A P I$ definition without requiring deep knowledge of the specification while (ii) keeps the modeling environment simple and manageable; and (iii) allows staying compliant with the AsyncAPI Specification for those unfamiliar with modeling (iv) also enabling experienced developers and architects to refine and complete details of the architecture that cannot be captured using Ecore in an easy way ${ }^{8}$.

In order to integrate data models in the proposed development workflow, we have defined both the Ecore to AsyncAPI model-to-model (M2M) and the AsyncAPI to JSON M2T transformations shown in Fig. 3: the former transforms an annotated data model to its equivalent AsyncAPI model, while the latter serializes back the abstract definition of the AsyncAPI model to its JSON-based representation. Once we have both an AsyncAPI model and its JSON-based representation, the workflow can proceed as explained in Sect. 4.1.

Example An architect willing to use AsyncAPI as the single source of truth in our warehouse use case would proceed as follows. The architect would create a data model of the messages exchanged among all the devices in the messagedriven architecture. After adding the desired annotations for the servers, channels, and messages, an equivalent AsyncAPI model-which is automatically transformed to the corresponding AsyncAPI JSON definition-is generated ${ }^{9}$. If we consider only the monitoring part of our use case, in practice, this definition is exactly the one we show in Listing 2 since our proposal fully complies with the AsyncAPI Specification. While the user is editing, our AsyncAPI Toolkit creates the corresponding AsyncAPI model and executes the AsyncAPI to code M2T transformation generating the internal DSL on-

\footnotetext{
7 We use the term bootstrapping because we transform a less expressive definition - an annotated Ecore data model — to a more expressive definition - the AsyncAPI JSON definition - which will act as the new starting point of the development process. In this sense, and for the sake of simplicity, once an AsyncAPI JSON definition has been created, modifications on it cannot be ported back to an Ecore data model because of its different levels of expressiveness.

${ }^{8}$ Alternatively, data modeling in the AsyncAPI Toolkit could be achieved by providing a UML profile to annotate UML class diagrams. While this possibility has not been yet discarded and can be considered complementary, for the current purposes plain annotations have been considered enough.

${ }^{9}$ Of course, the architect can start the development process by directly creating the AsyncAPI JSON definition, but as aforementioned, starting from data models allows bootstrapping the development process with less effort as we justify in Sect. 6.3.3.
} 
the-fly. The generated DSL-which is an executable library exposing a fluent API in Java-can be directly distributed in source code form or as packaged binaries to the developers of the different components of the architecture (e.g., the IoTBoxes, the cloud, or the frontend). Thus, a developer wanting to publish a message or consuming a message does not need to care about other elements in the architecture or external documentation: all he or she has to do is to import the libraries of the DSL. The DSL will provide all the functionality needed to connect to a specific broker, create a specific message in the right format, and publish it, or vice versa: connect to the specified broker, subscribe to a specific topic, and receive the messages in the right and native format of the platform being used.

\section{The AsyncAPI Toolkit under the microscope}

We have implemented the AsyncAPI Toolkit workflow as an open-source solution, ${ }^{10}$ and as highlithed above, we have followed model-driven development principles to create it. Instead of manually developing a set of editors supporting the textual AsyncAPI JSON Grammar or the AsyncAPI metamodel, we have chosen Xtext to provide both a concrete and an abstract syntax for AsyncAPI. Furthermore, we take advantage of all the EMF ecosystem to develop the rest of the toolkit. This is the case for our proposal for message modeling-which uses existing Ecore editors-and all the M2M and M2T transformations shown in Fig. 3, namely Ecore to AsyncAPI, AsyncAPI to JSON and AsyncAPI to code.

Next, we describe in detail how our AsyncAPI Toolkit has been built ${ }^{11}$ and how architects and developers can take advantage of it.

We will start by explaining both the AsyncAPI JSON grammar in Xtext and the automatically generated AsyncAPI metamodel, since these are central assets of our proposal. Next, we will explain how to bootstrap an AsyncAPI JSON definition from an Ecore-based data model. And we will end the Section by explaining how the fluent API providing the internal DSL is generated and used.

\footnotetext{
$\overline{10}$ https://hdl.handle.net/20.500.12004/1/A/ASYNCAPI/001.

11 Only short illustrative excerpts of the different elements of the AsyncAPI Toolkit will be shown for brevity purposes. For a full reference, please check our repository.
}

\subsection{A JSON-based concrete syntax for AsyncAPI}

The main manual step that Xtext requires to provide a concrete syntax for a textual language is the development of an Xtext grammar. Listing 3 shows an excerpt of the grammar ${ }^{12}$ we have defined to support the definition of AsyncAPIs in JSON following the concrete syntax proposed in [5].

In short, we have defined an Xtext rule for each one of the concepts defined in Schema section of the aforementioned document. Listing 3 shows-in a simplified way-the rules to define in JSON an AsyncAPI specification version 2, with its Info, a set of Servers, Channels, and the Components Section. Taking as an example the AsyncAPI rule (line 1), in line 2 we specify that the application of the rule will produce an AsyncAPI object when parsing an input text, while lines 3 and 12 specify that a AsyncAPI is a textual element enclosed between the characters $\{$ and $\}$. The parentheses in lines 4 and 11 denote an unordered group, i.e., the patterns between them, which are separated by an \& symbol, may match only once and in any possible order. Line 5 , for example, specifies that the version of an AsyncAPI is a sequence of characters starting with the "asyncapi " keyword, followed by a : symbol and followed by a text matching the VersionInfo rule (which in turn, is an enumerated, specifying that only version "2.0.0" is supported). The value of the parsed version number will be stored in an attribute of the AsyncAPI object named version of type VersionNumber. It is necessary to clarify some details of the grammar: first, in order to get advantage of the features provided by the Xtext unordered groups, we have defined the commas between groups as optional $\left({ }^{\prime}, '\right.$ ? expression near the end of each group), and second, we have relaxed the requirements of some elements marking them as optional (? symbol at the end of each group) to minimize the number of errors reported while parsing the input files for not overwhelming the users of the tool ${ }^{13}$. These optionalities can be, however, later enforced programmatically so that the tool only accepts valid JSON instances.

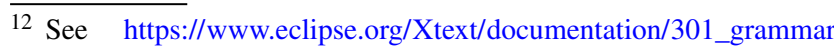
language.html for a full reference of Xtext grammars.

13 Since Xtext performs a live validation of the document being edited, it is necessary to allow inconsistent intermediate states of the underlying model while creating it: if hard requirements are set at the metamodel level, most of the errors reported will refer to parts of the document not added yet, rather than to real errors of the already written parts.
} 
Listing 3: Excerpt of the Xtext grammar

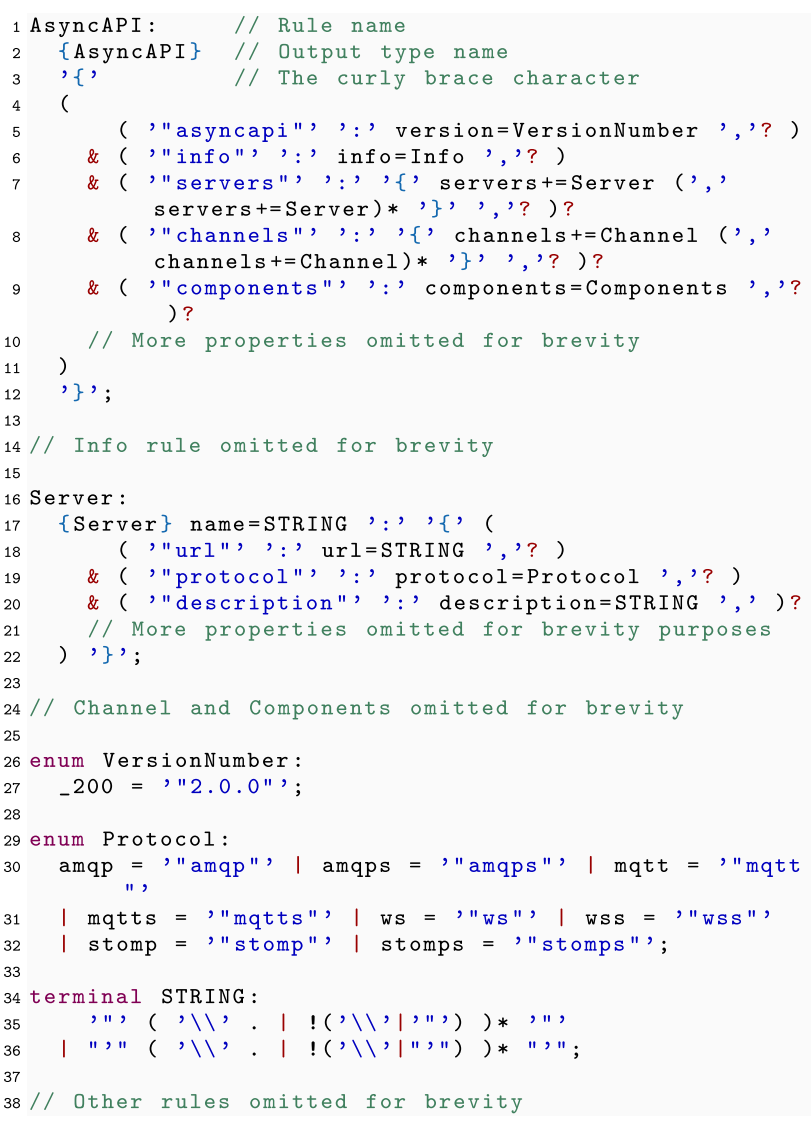

Example As it can be seen, the AsyncAPI definition included in Listing 2 can be parsed by applying the rules of the grammar showed in Listing 3: the curly brace in line 1 of Listing 2 matches the curly brace in line 3 of Listing 3 ; the tokens in line 2 of Listing 2 ("asyncapi" : "2.0.0") match the tokens specified in line 5 of Listing 3; etc.

\subsection{An abstract syntax for AsyncAPI}

Based on the grammar above, Xtext is able to generate an equivalent EMF-based metamodel. Figure 4 shows the Ecore metamodel generated as a result of the AsyncAPI JSON grammar. As aforementioned, instances conforming to this metamodel are automatically created out of textual descriptions thanks to the tooling generated by Xtext. As it can be observed, it basically contains a class for each one of the rules defined in the Xtext grammar, and each one of the classes contains the attributes specified in its corresponding rule. For example, among other elements, Fig. 4 shows the AsyncAPI and Server classes which correspond to the rules included in Listing 3, as well as the enumerations for VersionInfo and Protocol. The existence of this automatically generated metamodel enables the use of any other EMF-based tool, such as model transformation engines.
It is noteworthy to mention that we have been extremely careful when defining the AsyncAPI JSON grammar so that the generated EMF-based metamodel closely represents the domain-i.e., the AsyncAPI Specification - and not only its textual representation. Thus, this metamodel is not only an utility artifact that enables us to parse AsyncAPI JSON specifications, but it is a meaningful metamodel close to the concepts of the AsyncAPI Specification: as it can be seen, there is a direct match between it and the main concepts explained in Sect. 3, and as a consequence, we will not describe in detail the concepts shown in the figure to avoid being redundant.

Example The result of parsing the AsyncAPI definition in Listing 2 is partly shown in Fig. 5. As it can be seen, the automatically generated model contains a single root AsyncAPI object, containing Info, Server, Channel and Component objects. The properties of the production Server are shown in the Properties tab. As the AsyncAPI definition in Listing 2 specifies, the name of the Channel object is iotbox/\{id\}/monitor, and it contains the operations publishstatus and subscribestatus, and the Parameter id. The Components object contains the Schemas pathInfo and beltInfo, and the Message statusMessage.

\subsection{An Ecore-based concrete syntax for data modeling to bootstrap a message-driven architecture}

As it can be seen in Listing 2, the specification of the messages format is the longest and most verbose part of a message-driven architecture definition, even for the simplest use case. This is even more evident in real-world scenarios, where we can find tens or hundreds of different messages in the same architecture. For this reason, we propose to allow bootstrapping an AsyncAPI-based development process by modeling the different messages that may be sent and received. Since Ecore is the core (meta)modeling language provided by EMF - also having a stable and adequate tool support-we have decided to adopt it as the most convenient way to provide data modeling capabilities in our AsyncAPI Toolkit. In short, Ecore can be considered as an implementation of the Essential MOF (EMOF) Model proposed by the OMG [40].

Figure 6 shows an excerpt of the Ecore (meta)modeling language ${ }^{14}$. In the figure, classes with a gray background are abstract, while classes with a lighter yellow background are concrete. As it can be seen, Ecore is a constrained subset of the UML2 [38] class models-as EMOF also is.

\footnotetext{
${ }^{14}$ Since the AsyncAPI Toolkit focuses on data modeling, Fig. 6 only focuses on structural modeling primitives. Other primitives-such as EOperations - have been omitted for brevity purposes.
} 


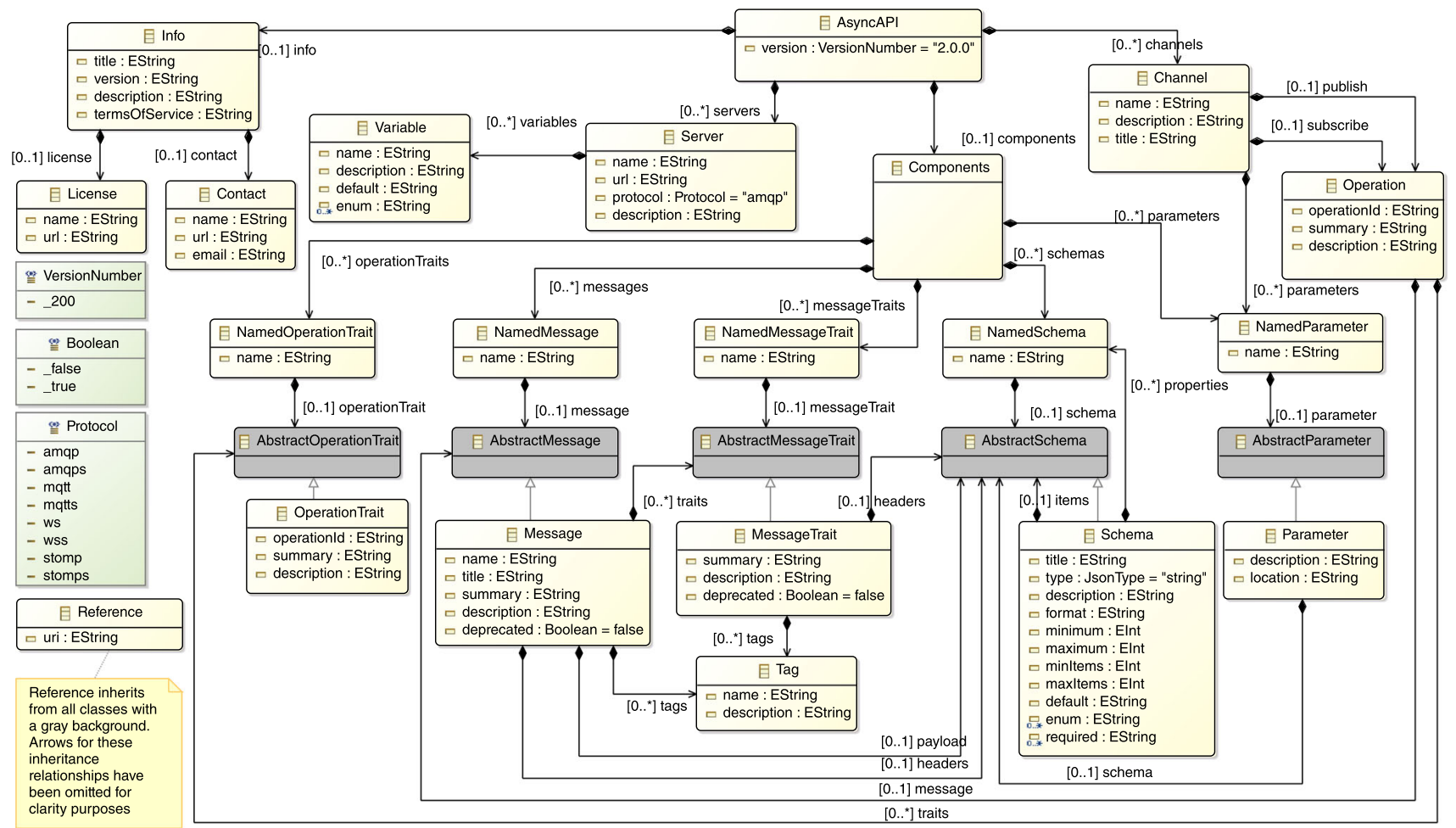

Fig. 4 AsyncAPI metamodel

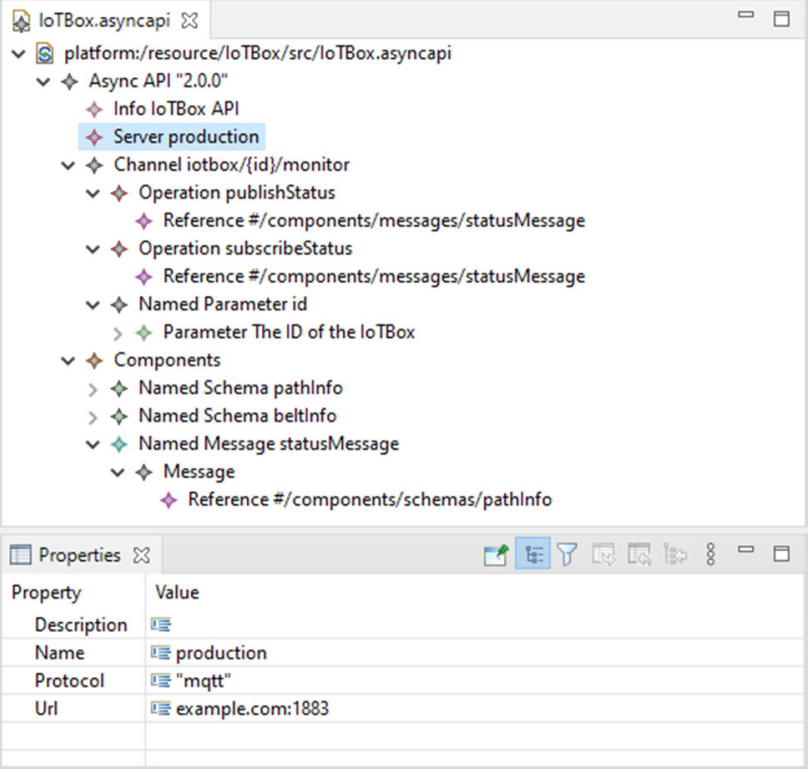

Fig. 5 AsyncAPI definition of the factory use case represented as an instance of the AsyncAPI metamodel

In short ${ }^{15}$, an Ecore model is composed of EModelElements, which can be either elements with a name (ENamedElements) or annotations (EAnnotations). Among the

$\overline{15}$ For an extensive explanation of Ecore, see [49].
ENamedElements we find: EPackages, EClassifiers, ETypedElements and EEnumLiterals. EPackages are used to group EClassifiers. EClassifiers, which can be either EClasses and EDataTypes, are used to represent and classify domain entities. ETypedElements - and EStructuralFeatures - are used to represent properties of domain entitites via EAttributes, or relationships among domain entities via EReferences. Regarding EAnnotations, any EModelElement can have any number of them. EAnnotations are identified by its source attribute and contain a set of details in the form of a key-value map. In Ecore, EAnnotations have been used since the beginning as the standard way to customize automated processes which take as an input a model ${ }^{16}$. Thus, in AsyncAPI Toolkit we have adopted this same approach to complement data models with additional information that will allow obtaining a fully working AsyncAPI definition.

Table 1 shows the annotations currently supported by the AsyncAPI Toolkit. Information about Servers is directly added to the EPackage containing the data model. There can be as many Server annotations as servers are in the architecture. For each server, we can specify its name, its url-including the listening port—and the supported protocol. Details about Channels, Messages, and Schemas can be all added in the corresponding EClass. This is because: (i)

\footnotetext{
${ }^{16}$ For example, EAnnotations are used to include model constraints that can be checked at runtime. EAnnotations are also used to tweak the behaviour of the standard code generation templates.
} 


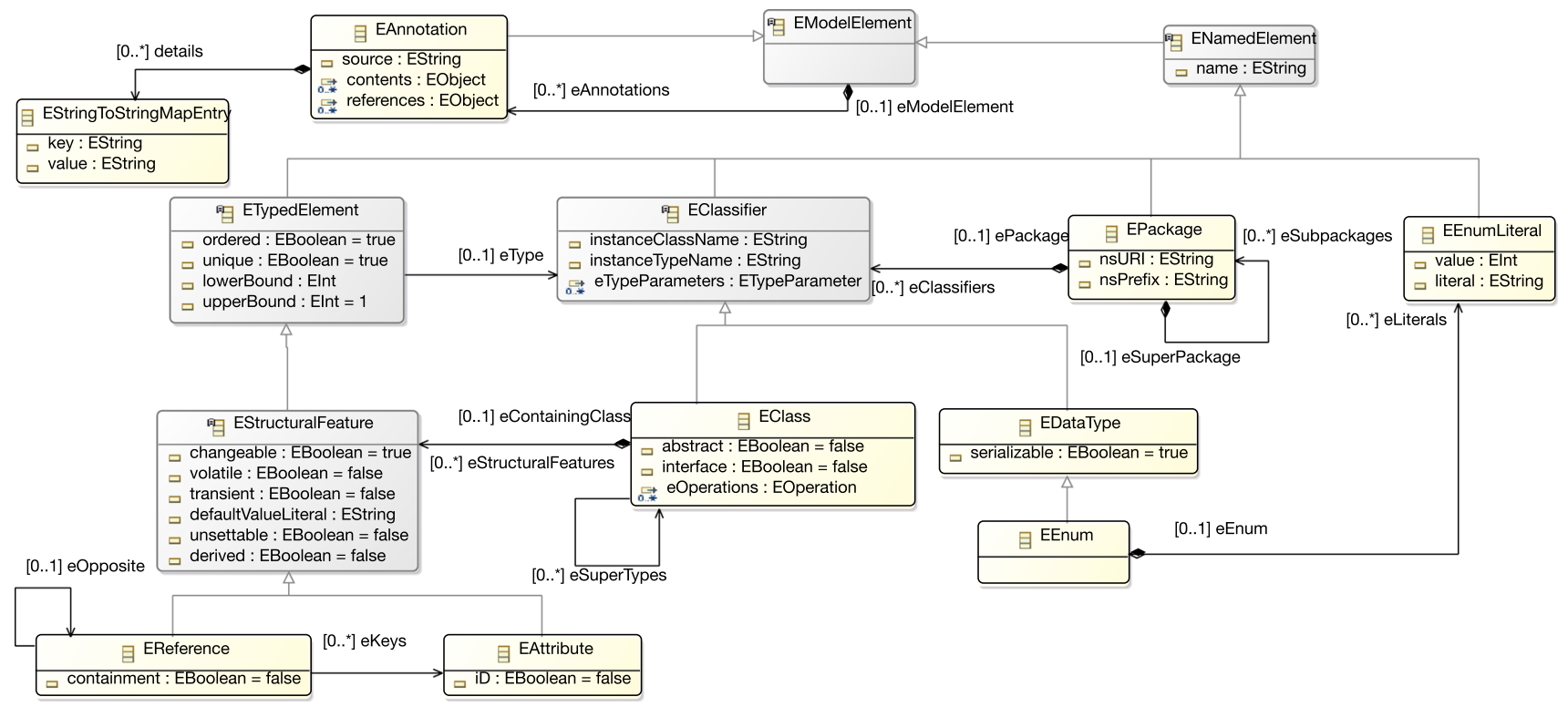

Fig. 6 Subset of the Ecore metamodel

Table 1 ecore annotations

\begin{tabular}{|c|c|c|c|}
\hline ECore element & EAnnotation source $^{a}$ & description & keys \\
\hline Epackage & Server & list of servers & $\begin{array}{l}\text { name: server name } \\
\text { url: server url, including port } \\
\text { protocol: Asyncapi supported protocol }\end{array}$ \\
\hline Eclass & Channel & $\begin{array}{l}\text { the eclass represents the pay- } \\
\text { load of a given channel }\end{array}$ & $\begin{array}{l}\text { description: channel description } \\
\text { publish: publish operationid } \\
\text { subscribe: subscribe operationid } \\
\text { parameters: comma-separated list of parameters used in the channel } \\
\text { name, if any }\end{array}$ \\
\hline Eclass & Message & $\begin{array}{l}\text { the eclass represents the pay- } \\
\text { load of a given message }\end{array}$ & name: message name \\
\hline Eclass & Schema & $\begin{array}{l}\text { the eclass represents a pay- } \\
\text { load }\end{array}$ & $\begin{array}{l}\text { name: schema name } \\
\text { title: friendly name of the schema }\end{array}$ \\
\hline EStructuralfeature & Schema & $\begin{array}{l}\text { the estructuralfeature is a } \\
\text { property part of a payload }\end{array}$ & title: friendly name for the property \\
\hline
\end{tabular}

\footnotetext{
${ }^{a}$ to avoid redundancies, we have omitted the common part of the eannotation source-an identifier in the form of a uri, which in this case is http:// io.github.abelgomez/asyncapi/eannotations/—for all elements in the table (e.g., server should be read as http://io.github.abelgomez/asyncapi/ eannotations/server)
}

EClasses represent domain entities, which in our case are Messages and their corresponding payload (i.e., Schema), and (ii) a single Channel refers to a single Message-for both its publish and subscribe operations-and a single Message refers to a single Schema.

As a consequence, all this information can be added in the EClass itself. For a Channel, we can specify its name, a description, the publish and subscribe operationIds, and the parameters, if any; for a Message, we can specify its name; and for a Schema we can specify its name and its title. It is noteworthy to mention that most of these annotations are not strictly required ${ }^{17}$. This may happen for two reasons:

(i) because a default value can be directly derived from the plain Ecore model; or (ii) because the property we are specifying is optional in the AsyncAPI Specification.

The former is what happens when an EClass is annotated as a Channel, and no Message nor Schema annotations are

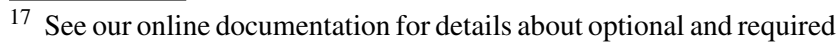
annotations.
} 


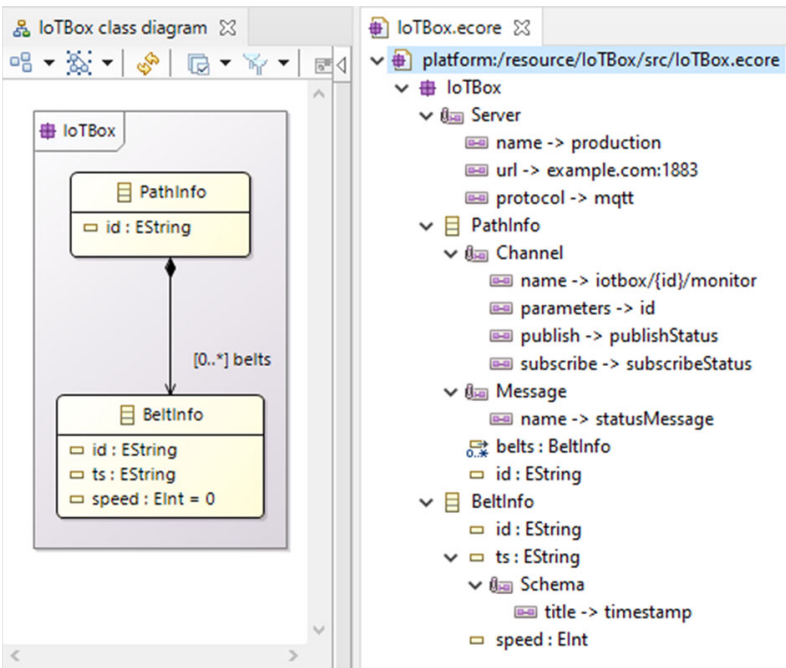

Fig. 7 Ecore representation of the IoTBox example

added: in that case, Message and Schema names are derived from the EClass name). An example of the latter case is the Schema EAnnotation when applied to an EStructuralFeature: title is optional in the AsyncAPI Specification and thus is not required.
Example Figure 7 shows what the messages of our running example look like whenmodeled using Ecore. The left-hand side of the figure shows how the data model is represented in a graphical way, while the right-hand side of the figure shows the same model represented in a tree viewer with the corresponding EAnnotations. As it can be seen, we have a single EPackage called IоTBox which contains two EClasses: PathInfo and BeltInfo. PathInfo has an id EAttribute and contains a set of BeltInfos via the belts EReference. A BeltInfo has three EAttributes: id, ts, and speed. This data model perfectly matches the JSON Schema expressed in lines 45-77 of Listing 2.

In the right-hand side of the figure, we have included the Servers information directly in the IoTBox EPackage. Since statusMessages are published and subscribed under topic-i.e., Channel - iotbox/ $\{i d\} / m o n i t o r$, and pathInfo is the Schema of statusMessage, we have added the corresponding Channel and Message EAnnotations to the PathInfo EClass. Since the name of the PathInfo EClass matches ${ }^{18}$ the pathInfo Schema name, there is no need to add the Schema EAnnotation. Finally, the Schema annotation in the ts property of BeltInfo indicates that we want to refer to this property as timestamp rather than $t s$.

Listing 4: Excerpt of the Ecore to AsyncAPI M2M transformation

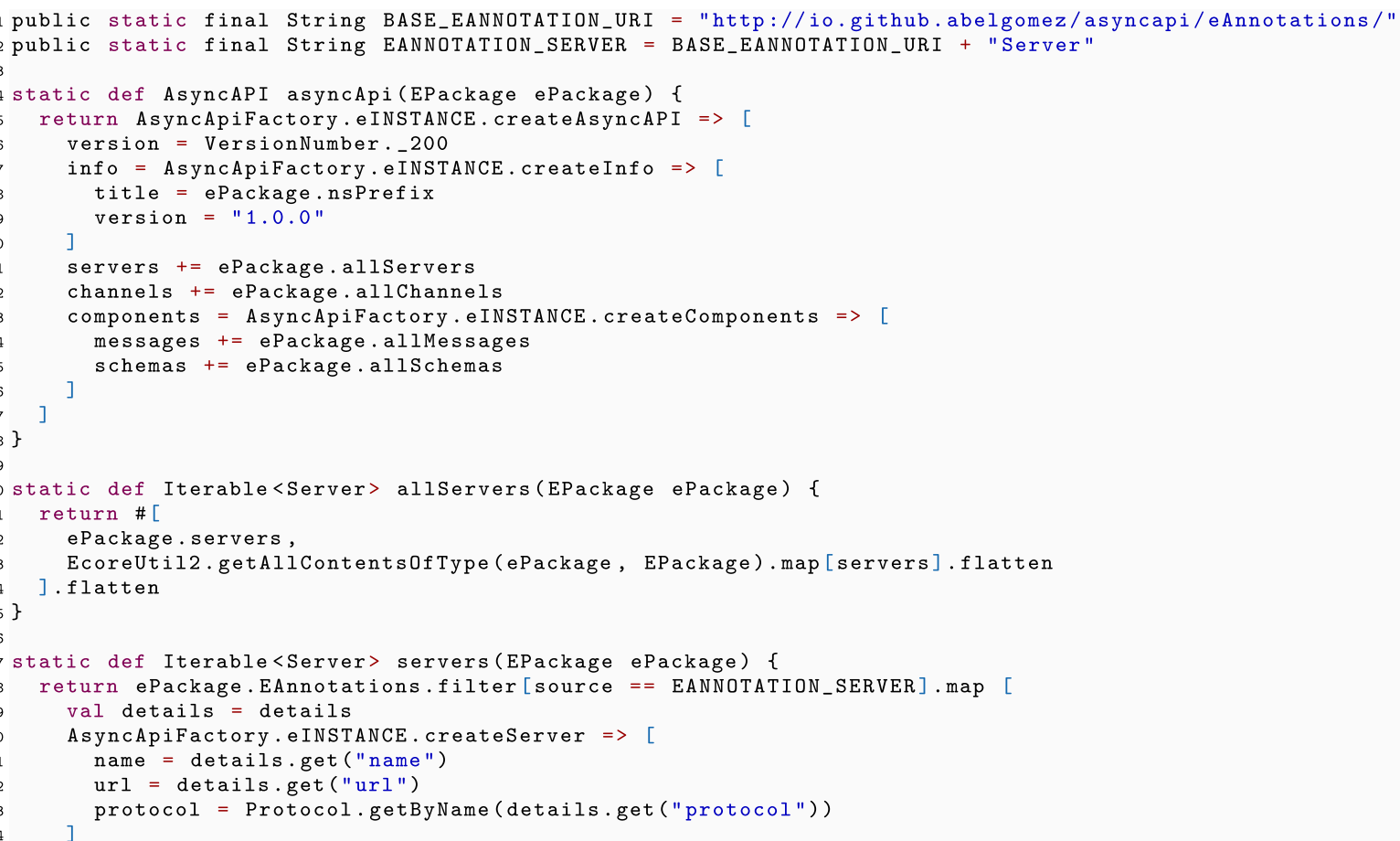

$\overline{18 \text { Letter case }}$ conventions differ between Ecore EClasses-where names must start by an uppercase letter-and JSON Schemas-where names usually start by a lowercase letter. As a consequence, the name PathInfo in Ecore is considered equivalent to pathInfo in JSON. 


\subsection{From an annotated data model to an AsyncAPI definition}

Once an annotated data model has been created, it can be automatically transformed to a valid AsyncAPI definition. As depicted in Fig. 3, this transformation is done in two steps: first, the Ecore to AsyncAPI M2M transformation converts the annotated model into an AsyncAPI model, and second, the AsyncAPI to JSON M2T transformation serializes the AsyncAPI model into a JSON document.

To implement both transformations, we have used Xtend [52]. Xtend is a flexible and expressive dialect of Java, which compiles into readable Java compatible source code. Xtend is the language which provides code generation support to the Xtext framework, and as a consequence, transformations in this language can be very easily integrated in our AsyncAPI Toolkit.

Listing 4 shows an small excerpt of the Ecore to AsyncAPI M2M transformation. Lines 4-18 show the rule to create an AsyncAPI object from an EPackage object. Specifically, in line 5 we specify that we will return a new AsyncAPI object created by calling the factory method createAsyncAPI. Then, we make use of the with operator $(=>)$ to initialize this new object by executing the code between square brackets. Code between square brackets is a lambda expression that is executed over the left-hand-side argument of the with operator returning it. Thus, version, info, servers, channels and components are not local variables, but properties of the new AsyncAPI object. This pattern to create and initialize objects is repeated throughout the whole transformation for its simplicity, readability and conciseness (e.g., see lines 7-10, 13-16 and 30-34). Line 6 shows a simple initialization of the AsyncAPI version property from a static value (VersionNumber is an enumeration, and _200 a literal); lines 7-10 and 13-16 show the initialization of properties with nested objects (an Info object and a Components object, respectively); line 8 shows the initialization of a property from a primitive value extracted from the source Ecore model; and lines 11, 12, 14 and 15 show the initialization of properties by delegating the creation of the target object to other transformation rules. In this case, two important features of Xtend are exploited: extension methods and infix operators and operator overloading. Extension methods allow adding new methods to existing types without modifying them: when invoking a method that can receive arguments, instead of passing the first argument inside the parentheses of the method invocation, the method can be called with the first argument as its receiver. Infix operators together with operator overloading allow Xtend to express the add operation in a collection as $+=$. Considering these two features, we can see that servers $+=$ ePackage.allservers is indeed equivalent to servers. addAll (allservers (ePackage)).
Method allservers applies the servers method to the EPackage passed as an argument and all its nested EPackages. The former calculation is done in line 22, while the latter is done in line 23 (the . map [ . . ] function allows applying the operation passed as argument to all the elements of a given collection). Then, an immutable list is created with the result of both transformations via the \# [ . . . ] operator, and this list is finally returned after being flattened (lines 21-24).

Finally, the servers method (lines 27-36) creates a list of Servers from a given EPackage. To do it, first we extract all EAnnotations in the EPackage whose source is Server, and afterwards, for each one of these EAnnotations, we create a new Server from its details ${ }^{19}$.

The rest of the model is created by executing the transformation rules in lines 12, 14 and 15-which, in turn, call other transformation rules.

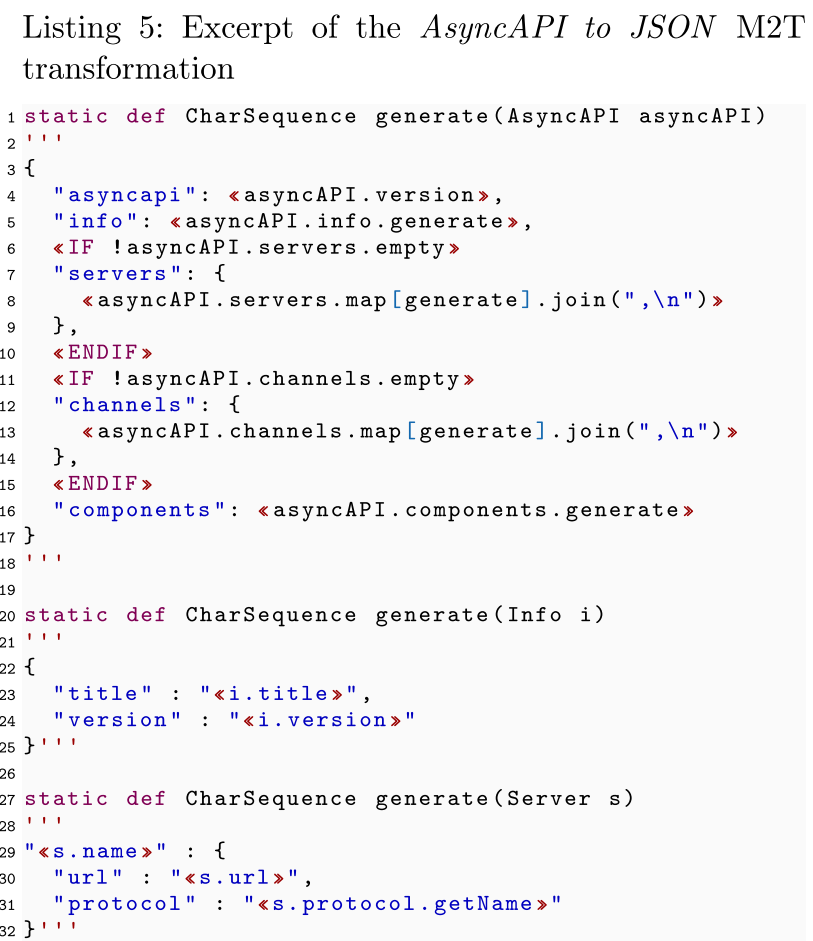

Once an AsyncAPI model has been obtained, serializing it to its JSON-based representation is straightforward. In order to define the AsyncAPI to JSON M2T trasnformation, we have used Xtend again, but now taking advantage of its template expressions. Templates are surrounded by triple single quotes (' ' ' ) and allow for readable string concatenation. A template expression can span multiple lines, and expressions can be nested using guillemets-i.e., «expression».

\footnotetext{
19 We must assign the details property of the EAnnotation to a local variable (i.e., val details = details in line 29) because the details property is not available in the scope of lines 31-33 (which is Server, and not EAnnotation).
} 
Listing 5 shows the transformation rules that serialize the AsyncAPI (lines 1-18), Info (lines 20-25) and Server (lines 27-32) objects. In this case, notice that we also make use of extension methods to improve the readability of the transformation. As aforementioned, text enclosed between three single quotes-with the exception of the bits enclosed between guillemets «...»-is copied as is in the final textual file. Text between guillemets is evaluated, and its tostring ( ) representation is automatically inserted at the expression position. It is noteworthy that templates allow conditions as shown in lines $6-10$ and $11-15$. In those cases, the text enclosed between the IF and ENDIF markers will only be serialized if the condition is met.

Example By applying the Ecore to AsyncAPI M2M transformation, we are able to transform the data model depicted in Fig. 7 into the AsyncAPI model depicted in Fig. 5. As it can be seen, the rules shown in Listing 4 would allow to create the AsyncAPI, Info and Server objects shown at the upper part of Fig. 5 out of the EPackage declaration in Fig. 7. Afterward, after applying the AsyncAPI to JSON M2T transformation, the AsyncAPI model in Fig. 5 can be serialized as it is shown in Listing 2. For example, the transformation rules shown in Listing 5 are the ones that generate lines $1-12$ and 80 of Listing 2: lines 3 and 17 in Listing 5 produce lines 1 and 80 in Listing 2; line 4 in Listing 5 produces line 2 in Listing 2; lines 5 and 20-25 in Listing 5 produce lines 3-6 in Listing 2; and lines 7-9 in Listing 5 produce lines 7-12 in Listing 2.

\subsection{An internal Java DSL for effective message-driven communication}

The last step of our proposed workflow is the generation of a library implementing an internal DSL, which can be used and shared among all the elements in the infrastructure, and that will ensure that all participants know the same topics and are able to create and consume messages conforming the same schemas and formats. Currently, our AsyncAPI Toolkit only supports the generation of Java code, but other languages and technologies can be easily plugged in by providing the proper code generation templates.

The M2T transformation applies the following rules:

A Server is chosen as the default Server of the architecture. Channels are transformed to packages whose name corresponds to the Channel-or topic —name. Hierarchies of topics-i.e., using slashes (/)-are respected.

Operations in Channels are transformed to classes inside the packages of their corresponding Channels. Classes for channel Operations, among other things, provide static operations for publishing and subscribing in their respective topics using the default Server. If Channel permits the use of Parameters, a nested class is generated to manage the parameter substitution and recovery.

Messages do not generate a Java artifact: since there is a direct correspondence between Messages and Schemas (via the payload association), information about Messages is integrated within the corresponding Schema when needed.

The Components object is transformed to a components package.

Schemas are transformed to immutable classes: they are cloneable and serializable (via Gson ${ }^{20}$ ), have getters but no setters, and have a private constructor. Instances are created via a dedicated nested builder class.

Schema classes can be generated directly in the components package if they are reusable, or nested. For example, an Operation can have nested declarations for its corresponding Message and Schema. In that case, the Schema class is declared as a nested class within the Operation class.

Listing 6: Excerpt of the code generation template

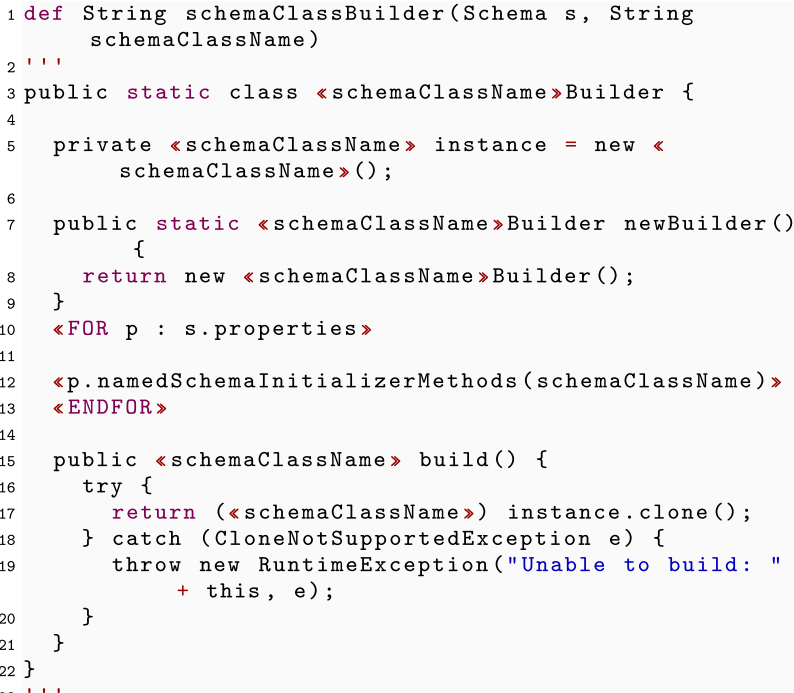

Listing 6 shows a very small excerpt of the code generation template. Specifically, it shows the template method generating the builder in charge of creating instances of a Schema class (which, when serialized, will be the payloads of the messages sent and received). As shown in line 1, this method receives as an input the Schema instance as declared in Fig. 4, and the Schema class name ${ }^{21}$. As it can be seen in line 3, the template generates a class, whose name is the one of the

\footnotetext{
${ }^{20} \mathrm{https}: / /$ github.com/google/gson.

21 This is because JSON Schemas do not have a name, as a consequence, the class name has to be calculated from other elements, such as the map containing it, the Message for which it is a payload, the Operation sending or receiving it, or any other element of the architecture.
} 
Schema class plus the suffix Builder. The builder contains a private instance of the Schema class (line 5), which will be the one being modified and returned to the client code once the build process finishes. The builder class contains a static factory method (newBuilder ( ), lines 7-9), and a builder method (build ( ), lines 15-21) that finishes the build phase and returns a immutable copy of the instance of the Schema class. As it can be seen in lines 10-13, the template generates an initializer method for each one of the properties of the schema. These are the methods that the client code must call between the factory method and the build method: since the builder class is nested within the Schema class, and the Schema class does not have setter, these initializer methods will be the only ones allowed to initialize and modify the instances of the Schema class. After that, they will remain immutable.

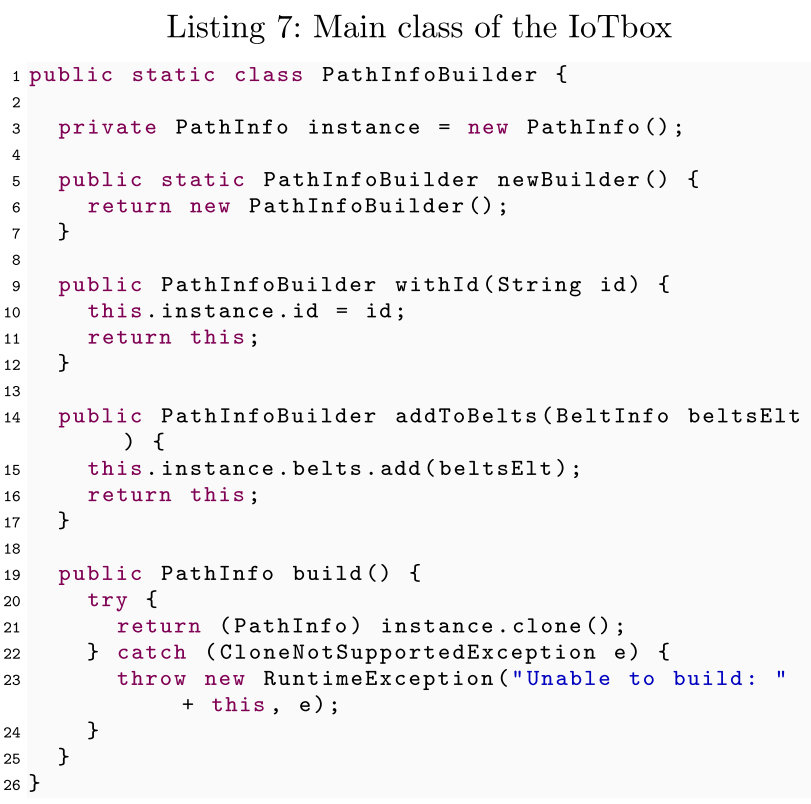

Example Listing 7 shows what the generated code for the builder class looks like for the pathInfo Schema. As explained in Sect. 3 and Listing 2, a pathInfo is an object with two properties: id, a string; and belts, an array of beltInfo objects. Thus, from the pathInfo Schema a PathInfo class is generated (not shown) with a PathInfoBuilder nested class. It can be seen that the generated code contains the private instance of the PathInfo (line 3), the factory method (lines 5-7), the builder method (lines 19-25), and two initializer methods. The first initializer method (lines 9-12) is in charge of setting the id of the PathInfo, which is a Java String. Since id is single-valued, the initializer method is prefixed with the word with. The second initializer method (lines 14-17) is in charge of initializing the list of BeltInfo objects. Since belts is many-valued, the initializer method is prefixed with the words addTo.
As it can be seen, the library provides specific artifacts for the most important concepts of the AsyncAPI definition:operations on each channel-topic — are unequivocally grouped in their specific packages; payloads of messages can only be created by using specific builders; and messages can only be sent and received using dedicated methods of specific classes.

Forcing developers to use builder classes and getters of the generated Schema classes-among other things—guarantees that the structure and the type of the messages are preserved and shared among all the elements of the architecture. The same applies, for example, with respect to the classes for publishing and subscribing since the relationship between Channels, Operations, Messages and Schemas is explicitly encoded in the internal DSL: static typing in Java ensures that developers do not mix incompatible types, or publish (or receive) messages in the wrong topics ${ }^{22}$.

Example Listings 8 and 9 show two examples of client code using the internal DSL in Java generated for our example AsyncAPI definition (Listing 2), and more specifically, Listing 8 shows the code needed to create and publish the example message shown in Listing 1. As it can be seen in the imports (lines 5-6), there exists a iotbox._id_.monitor package (generated from the iotbox/\{id\}/monitor Channel), with a MonitorPublish class (generated from the monitorPublish operation). Since the iotbox/ $\{i d\} / m o n i t o r$ Channel has an id parameter, also a PublishstatusMessage Params is created in order to perform the substitution and recovery of parameter values. It can also be seen that reusable Schemas (pathInfo and beltInfo) have produced the corresponding classes in the schemas package (see lines $8-9)$.

Thus, in order to create the message, developers only need to use the provided classes. For example, to create an instance of PathInfo, a new PathInfoBuilder can be obtained by invoking PathInfo.newBuilder() (line 13), and then, it can be initialized by using the provided fluent interface [20] (e.g., methods withId and addToBelts). If any of the methods needs another object as an argumentsuch as for addToBelts-it can be created by using the corresponding builder as shown in lines 15-21. When a friendly name is available for a given property - for example, because a title was specified - the method provided by the fluent interface will use it instead of the actual Schema property name. Lines 18 and 25 are an example of this: timestamps are a property called $t \mathrm{~s}$, but the provided method is withTimestamp rather than withTs, thus making the code more understandable. Once all the properties have been

\footnotetext{
${ }^{22}$ In any case, these checks can be proactively done in the generated code following a fail fast approach in the case these features are not natively provided by the language of the generated code (e.g., dynamically typed languages).
} 
set, the build() method is invoked. It is noteworthy to mention that validation logic_-such as checking of required properties - could also be added in the build() method following a fail fast approach.

Listing 8: Main class of the IoTBox Publisher

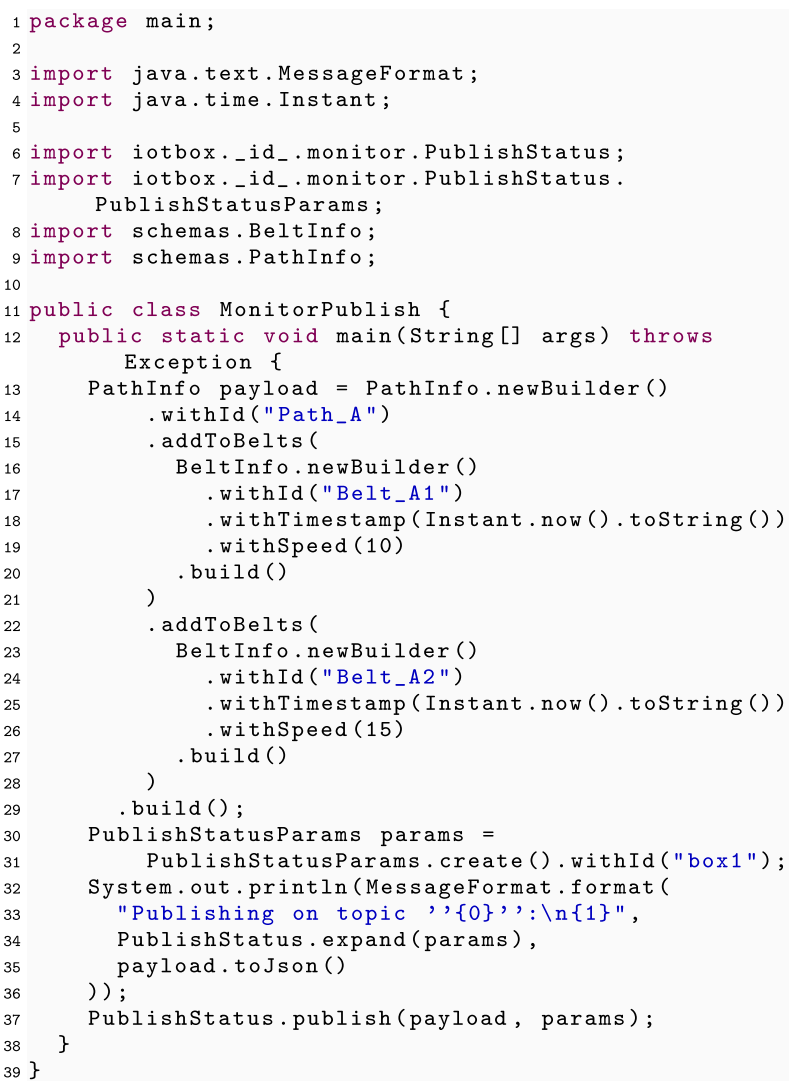

Listing 9: Main class of the IoTBox Subscriber

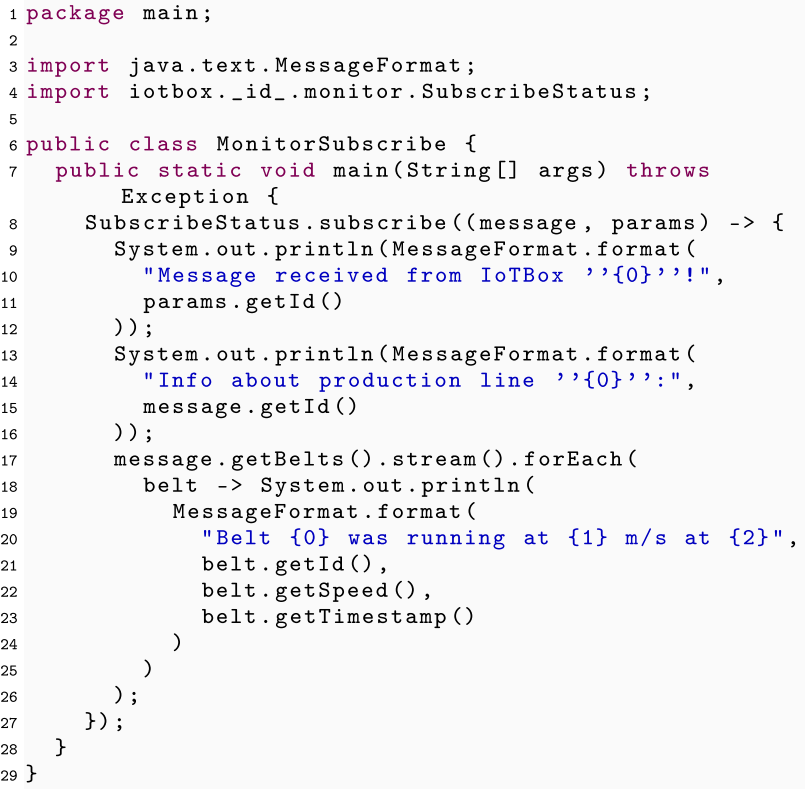

Finally, once the payload of the message and the parameters have been created-parameters are created using a similar fluent interface as shown in lines 30-31-the publish operation can be invoked (see line 37). The publish method of the MonitorPublish class only accepts instances of PathInfo as the first argument and instances of PublishStatusMessageParams as the second argument. The publish operation will be in charge of doing the parameter substitution, and publishing the payload passed as an argument in the iotbox/box1/monitor topic. This will ensure that both the payload and the topic names will be syntactically correct and will match.

Listing 9 shows how an example application-such as one running in the cloud-will subscribe to the iotbox/+/monitor topic and will receive messages sent to it. As it can be seen, it only needs to invoke the SubscribestatusMessage. subscribe method passing a callback function (expressed as a lambda expression in lines 8-27): the callback function will receive the value of the parameters in the params argument-which is of type SubscribeStatusMessageParams-and the message payload in the message argument-which is of type PathInfo. From this point on, client code can make use of the getters provided by the generated code to retrieve all the information from them. As it can be seen, the example code only prints all the received information by the standard output.

\section{AsyncAPI Toolkit Evaluation}

The aim of this section is to evaluate the AsyncAPI Toolkit. As we discuss in Sect. 7, there are no other tools focusing on the modeling of AsyncAPI definitions based on data models that allow generating fully functional executable code out-ofthe-box. Thus, we focus on evaluating the perceived usability and usefulness of our tool, and its strengths and weaknesses, based on short interviews with developers using AsyncAPI Toolkit in internal projects and with the results of an empirical experiment involving additional participants.

More specifically, the evaluation of the tool focuses on answering the following research questions:

RQ1 Which are the perceived benefits of using AsyncAPI Toolkit to define and develop an asynchronous message-driven architecture?

RQ2 Is it possible to use AsyncAPI Toolkit without previous knowledge of the tool? How much time is necessary to be autonomous and to obtain significant results, i.e., a running asynchronous architecture?

RQ3 Which are the weaknesses and strengths of AsyncAPI Toolkit? 
RQ4 Does using a graphical syntax for defining the AsyncAPI definition provide any benefits?

In order to answer these questions, we have conducted two different evaluations of AsyncAPI Toolkit. On the one hand, we have used AsyncAPI Toolkit internally in a number of projects and, as a result, a first set of conclusions and lessons learned have been drawn, as explained in Sect. 6.2, answering RQ1. On the other hand, an empirical external validation has also been carried out once the internal developers considered AsyncAPI Toolkit was mature enough. The aim of this external validation was twofold: first, quantifying the perceived benefits of using AsyncAPI Toolkit when developing a message-driven architecture, specially regarding learning and development time taking into account the previous experience of the subjects building similar projects by hand, and thus answering RQ2; and second, gathering feedback and educated opinions in order to answer RQ3 and RQ4. Details of this empirical evaluation are described in Sect. 6.3.

\subsection{Threats to Validity}

Before describing the evaluation, we first discuss the main threats to validity that can affect the result of the validations presented next. In this regard, the construct validity, internal validity, and external validity must be taken into account [56].

Regarding the construct validity, as described in the introduction, the aim of the AsyncAPI Toolkit is to help with the development of IoT projects. Therefore, to be able to evaluate the usability of the toolkit the main questions to be answered are related to the ease of use for developers of such projects. The four RQs are focused on evaluating exactly that, and in the following subsections the questions in the questionnaire are linked to the different RQs.

To be able to avoid bias in the internal validity, the weight of the evaluation is on the external validation. This is why although we developed a first internal validation focusing on our own experience using the AsyncAPI Toolkit in internal projects, an external validation was also done with people with different profiles and no previous involvement in the development of the toolkit.

The cornerstone of the performed evaluation is gathering relevant information from the subjects that conducted the experiment for external validity. The information was related to weaknesses and strengths, users impressions about usability and the perceived usefulness of the presented toolkit in order to answer the previously mentioned research questions. The external evaluation was mainly focused on interviews and questionnaires, as it allowed to collect relevant data from the subjects. As subjects are the basis of the evaluation, they also become the main threat.

Our threats are mainly associated with the participants, the number of subjects-which could have been increased to increase the soundness of the results-and the scales used for the measurements. In the First Phase, the subjects of our experiment could have a different previous knowledge before participating in the experiment. We tried to alleviate this issue in two ways: (i)we explicitly asked for this information in the survey to consider this variable in our experimentsalthough the claimed level of expertise is anyway subjective since each participant's scale may be different-and (ii) we tried to cover a diverse set of profiles in the selection by choosing engineers, developers, staff in training, researchers, etc., to gather different points of view.

On the other hand, since modeling tools used in the Second Phase were not developed as part of this experiment, we tried to focus the experiment on the contribution of the AsyncAPI Toolkit by selecting those subjects with previous expertise in MDD. As aforementioned, our idea is to avoid a bias toward the AsyncAPI Toolkit functionality caused by existing modeling tools, since non-expert users may be unable to distinguish preexisting tools from the AsyncAPI Toolkit itself.

Related to the scalability of the exercise to real-world problems, even though the example being developed in the evaluation phase was limited by the timing, it represented complex communication data that can be found in real world and that can be easily adapted to real scenarios.

\subsection{Internal Validation}

AsyncAPI Toolkit has been developed in the context of the MegaM@Rt2 ${ }^{23}$ project and it has already been applied to some of its use cases. It has also been tested in some internal projects at Ikerlan and it is being used for the development of a use case in the COMP4DRONES project ${ }^{24}$. This section aims to answer RQ1 (perceived benefits of the AsyncAPI Toolkit) and discusses some of the reflections and benefits observed during the development of these projects.

\section{Lower development and deployment time.}

Adopting the AsyncAPI Toolkit in a project significantly decreases the time to develop and deploy the software system. On the one hand, reusing the generic metamodel simplifies the definition of the schemas of the project and, with the automated code generation, there is no need to implement the boilerplate code in the client side, reducing manual timeconsuming tasks. In fact, our observations show that the time to develop the infrastructure code has been reduced to nearly one-third of the initial time. Obviously, there is an initial cost in developing the AsyncAPI Toolkit itself, training the people in using it, and adapting the continuous integration infrastructure so that all components share the same version of the library implementing the internal DSL. But this cost

\footnotetext{
23 https://megamart2-ecsel.eu/.

24 https://www.comp4drones.eu/.
} 
is quickly compensated when using it over several projects, as it happens with any new MDE infrastructure [14].

\section{Increased code quality.}

In addition to lowering the development time, the generated code is better structured and allows to reuse common code blocks, which increases the overall code quality. The reused parts are already tested; hence, the verification process of the system is simpler. Moreover, the generated code is also easier to maintain, as bugs or improvements in common parts need to be addressed only once. This leads to a reduction on engineering and maintenance costs.

In this sense, it has been detected that using the AsyncAPI Toolkit in different projects, the time to detect bugs has been decreased as more bugs are detected in the initial stages of the implementation and execution of the developed systems.

\section{DSL benefits for Industry 4.0.}

Another upside is that, as diverse existing application domains share similarities —especially regarding communication requirements - the same solution can be applicable to all of them. In this context, we can more easily port our Asyn$c A P I$-based solutions to a variety of related domains. This is especially interesting in the context of software product lines (SPLs).

\section{Easy documentation.}

AsyncAPI includes a tool to automatically generate documentation out of an API. Although it is not integral part of our toolkit, it can be directly used side by side the AsyncAPI Toolkit since we stick to the AsyncAPI Specification. Thus, from a model or definition created by the AsyncAPI Tool$k i t$, the AsyncAPI tool is able to generate the corresponding documentation in HTML, Markdown or React.

At Ikerlan, this feature has been regarded as an important aspect in the decision to move forward with the adoption of AsyncAPI Toolkit. This documentation capability, together with the use of AsyncAPI as single source of truth, enables all project participants (stakeholders, designers, architects, etc.) to share a common definition of the API, favoring interoperability and reducing the number of errors in the software development life cycle.

\section{Requirements definition, validation and maintainability.}

Being a message-driven architecture, capturing the requirements can be directly done with the AsyncAPI definition. Therefore, there is no need to maintain a separate text document that needs to be interpreted by developers, as the requirements are specified in the AsyncAPI definition itself.

This has been another key reason to select AsyncAPI for ongoing and future projects at Ikerlan. Combined with the previous point, we see AsyncAPI Toolkit as a toolkit able to support most phases of the development cycle, from requirements to code-generation to-in the future-testing.

\subsection{External Validation}

The following paragraphs will describe the process we have followed to conduct the external validation, describing in detail: (i) the experiment carried out; (ii) the set of samples; (iii) the threats; and (iv) the results or conclusions gathered from it.

\subsubsection{Experimental design}

The evaluation of the AsyncAPI Toolkit was carried out in two phases.

First Phase-For the first phase, 25 subjects were invited to implement with AsyncAPI Toolkit a small example of an industrial system that communicates asynchronously. The example used is the exact same one presented in Sect. 2: an automated warehouse with paths, belts and speed measurements, where monitoring data and configuration messages can be sent and received. To solve the exercise, the participants had to specify the complete architecture and the format of the messages in JSON, using the AsyncAPI Toolkit and following the AsyncAPI specification. After specifying the architecture, they had to implement two simple applications using the automatically generated classes based on the DSL-i.e., fluent API: one for publishing new messages and other for consuming messages. The participants were given a one-hour deadline to develop this exercise, knowing that the set time was tight. After trying to implement the example application, the subjects answered a questionnaire about their knowledge of the field, previous background, and their impressions on the use of the AsyncAPI Toolkit to solve the exercise. The goal of this first phase was to answer RQ2 and $\mathrm{RQ} 3$.

The questionnaire consisted on a set of questions listed in Table 2 that evaluate the performance and usability of AsyncAPI Toolkit. The questions aimed to assess the following aspects: (i) rate the previous knowledge; (ii) score the ease of use of the tool; (iii) evaluate the ease of installation; and (iv) measure development time. Questions \#1 to \#8 mostly aimed at answering RQ2. Questions \#9 to \#16 mostly aimed at rating the level of satisfaction with the different components and features of the tool, also including some open questions to gather additional suggestions or comments, and allowing us to dig deeper into the possible improvements thus answering RQ3.

Second Phase-After the first phase, results were analyzed and a second phase of the experiment was conducted based on them. This second phase consisted on evaluating the data modeling extensions of the AsyncAPI Toolkit-which are described in Sects. 4.2 and explained in detail in Sects. 5.3 and 5.4-with the goal of answering RQ4. Not all subjects participated in this second phase of the experiment: since modeling tools used in the Second Phase are part of the 
Table 2 Phase 1 questionnaire

\begin{tabular}{|c|c|c|}
\hline Question \# & Question & Possible answers \\
\hline \multirow[t]{7}{*}{1} & What is your previous knowledge on: & - \\
\hline & a) Java & 1 to 5 \\
\hline & b) Asynchronous communication & 1 to 5 \\
\hline & c) MQTT & 1 to 5 \\
\hline & d) Eclipse & 1 to 5 \\
\hline & e) AsyncAPI & 1 to 5 \\
\hline & f) MDD (metamodels, models, ...) & 1 to 5 \\
\hline 2 & How difficult has been to carry out the assigned task? & 1 to 5 \\
\hline 3 & How much time did you spend installing the AsyncAPI Toolkit? & Number of minutes \\
\hline 4 & How much time did you spend learning or acquiring the main concepts (knowledge of the subject)? & Open answer \\
\hline 5 & Did you finish the exercise? & Yes / No \\
\hline 6 & How much time did you spend implementing the example? (in case you finished) & Number of minutes \\
\hline \multirow[t]{7}{*}{7} & What did you manage to finish? (in case you didn't finish) & - \\
\hline & a) Implement config payload & Yes / No \\
\hline & b) Implement two config messages (publish and subscribe) & Yes / No \\
\hline & c) Implement monitor payload & Yes / No \\
\hline & d) Implement the two monitor messages (publish and subscribe) & Yes / No \\
\hline & e) Implement one of the applications (publish or subscribe) & Yes / No \\
\hline & f) Implement both applications (publish and subscribe) & Yes / No \\
\hline 8 & $\begin{array}{l}\text { How much time do you estimate you would require to finish the whole application? (in case you didn't } \\
\text { finish) }\end{array}$ & Number of hours \\
\hline 9 & What do you think about the tool? & 1 to 5 \\
\hline 10 & How intuitive and easy to learn would you say the tool is? & 1 to 5 \\
\hline 11 & Do you think the workflow of the tool is the correct one? & Open answer \\
\hline 12 & Would you add any feature to the tool? & Open answer \\
\hline \multirow[t]{7}{*}{13} & Please rate your level of satisfaction with the following aspects of The AsyncAPI Toolkit? & - \\
\hline & a) Ease of installation & 1 to 5 \\
\hline & b) Overall reliability & 1 to 5 \\
\hline & c) Overall performance & 1 to 5 \\
\hline & d) Completeness and organization of documentation & 1 to 5 \\
\hline & e) Clarity of documentation & 1 to 5 \\
\hline & f) Ease of updating the application & 1 to 5 \\
\hline 14 & $\begin{array}{l}\text { Considering your complete experience with the software, how likely would you be to recommend its use } \\
\text { to a friend or colleague? }\end{array}$ & 1 to 5 \\
\hline 15 & Do you think using the tool reduces development and deployment time? How? & Open answer \\
\hline 16 & Please add any additional comments/suggestions if you wish & Open answer \\
\hline
\end{tabular}

Eclipse environment and were not developed as part of this experiment, we focused on the contribution of the AsyncAPI Toolkit by selecting those subjects with previous knowledge in MDD-these were 11 subjects, almost half of the initial ones. We did this selection to avoid a bias toward the AsyncAPI Toolkit functionality caused by existing modeling tools. The exercise consisted in obtaining the AsyncAPI definition for the automated warehouse architecture by creating an annotated model of the messages of the system. For this, the subjects used Eclipse and the editor of their choice to create and annotate an Ecore model, generating the corresponding AsyncAPI definition with the AsyncAPI Toolkit.

Since the set of subjects was smaller and easier to manage than in the first phase, short interviews with the subjects were carried out during this phase. In order to guide these interviews, a list of predefined questions was specified. Interviews gave us the opportunity to better focus the questions and gather more valuable feedback. The questions guiding these interviews aimed at answering $\mathrm{RQ} 4$ are listed in Table 3. The answers given by the subjects were analyzed one by one, and grouping the same main ideas afterwards. Different details 
Table 3 Phase 2 interview question guide

\begin{tabular}{ll}
\hline Question \# & Interview Questions \\
\hline 1 & $\begin{array}{l}\text { What do you think about this feature [the graphical syntax] of the AsyncAPI Toolkit? } \\
\text { If you compare phase } 1 \text { way of implementing the AsyncAPI specification and this one, what advantages and disadvantages } \\
\text { do you find? }\end{array}$ \\
3 & $\begin{array}{l}\text { Do you think that using this feature to define the system facilitates the understanding of the whole system? Or on the contrary, } \\
\text { do you think the way used in the first phase is easier and more understandable? }\end{array}$ \\
4 & $\begin{array}{l}\text { Do you think that this feature adds value to the tool? How? } \\
\text { Do you miss something to be included in the tool? }\end{array}$ \\
6 & How would you rate this feature? And the whole tool? \\
\hline
\end{tabular}

that could suggest improvements or concerns were also considered for future work.

\subsubsection{Sample}

Participants were selected from the personnel—engineers, developers, staff in training-of Ikerlan and Universitat Oberta de Catalunya who responded positively to a personal invitation. In any case, no subject was directly or indirectly involved in the development of the AsyncAPI Toolkit or this proposal. The sample consisted of 25 subjects with very diverse knowledge in fields and tools like Java, asynchronous communications, MQTT, Eclipse, AsyncAPI or model-driven development (MDD). As listed in Table 2, their expertise level was captured in the questionnaire. A 1 to 5 rating scale was used to quantify their knowledge, where 1 means no knowledge and 5 means being an expert.

The chart in Fig. 8 depicts the participants' knowledge on the mentioned fields. As shown in the chart, subjects came from different backgrounds. Very few people had previous knowledge of the AsyncAPI specification, while for the rest of the fields, the previous knowledge was more evenly distributed.

\subsubsection{Results}

This section summarizes the results of the data collected with the questionnaire in the first phase and the guided interviews of the second phase.

First Phase-After analyzing the data, our experiment shows that the $36 \%$ of the subjects were able to complete the experiment by implementing the full use case, and for the remaining $64 \%$, another $36 \%$ estimated that would require only one additional hour. These time estimations are based on the subjects perceptions but also on real data. The exercise was divided in six small sections with similar difficulty and required development time. The $36 \%$ that estimated one additional hour to finish the exercise, did complete at least 3 sections, and more than half of them, completed 4 or 5 sections (as they stated in question number 7). The $20 \%$

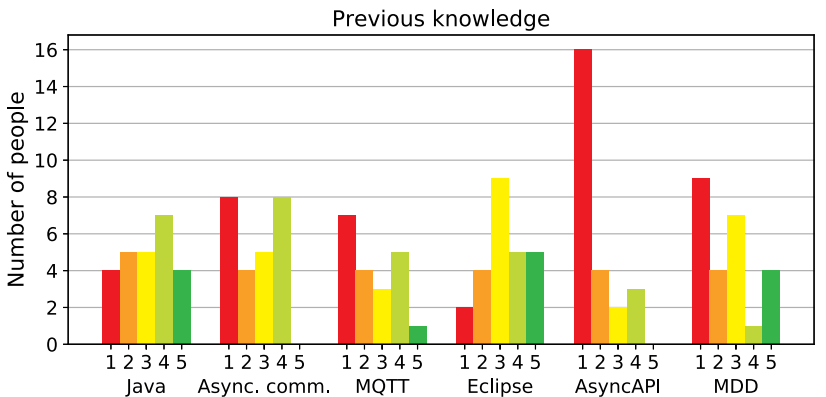

Fig. 8 Previous knowledge of the participants

of the subjects estimated between 2 and 4 hours while only the $8 \%$ thought would require more than 4 hours to complete the whole exercise. This reveals that only the $28.0 \%$ of the subjects estimated they would require more than 2 hours to finish the experiment. Taking into account the complexity of the exercise, as described above, we believe this is a very good result.

Looking at these percentages in detail, the data did not reveal a direct or close relationship between the subject's previous knowledge in a specific field and the time to complete the full use case. Subfigures in Fig. 9 present this data, where it can be observed that there is not a direct correlation between knowledge on the different specific topics with the time to finish, but more generally experienced participants were more successful. This is the case for Java (Fig. 9a), Asynchronous Communication (Fig. 9b), MQTT (Fig. 9c), Eclipse (Fig. 9d) and AsyncAPI (Fig. 9e). However, we detected an exception with the subjects that had previous knowledge in MDE, as they were more comfortable, and their first example implementation was more successful than people with little or no knowledge. Figure 9f confirms this trend. Those with little or no knowledge of MDE required more time to develop the use case (or estimated so), while those with more expertise finished or were close to finish in the fixed time.

What our experiment also confirms - thus answering RQ2 (previous knowledge and learning time)_-is that the learning curve is smooth: as Fig. 11 shows, the training to learn the AsyncAPI Toolkit basics is not specially long or hard. 


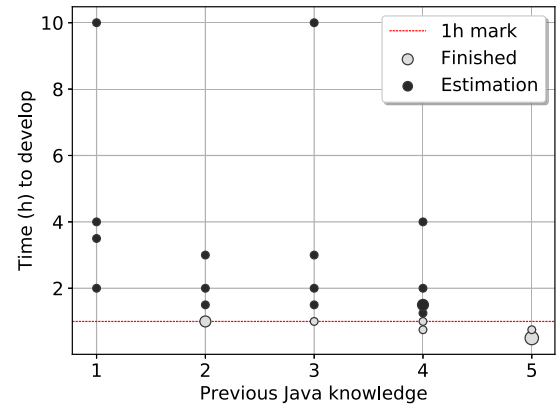

(a) Java

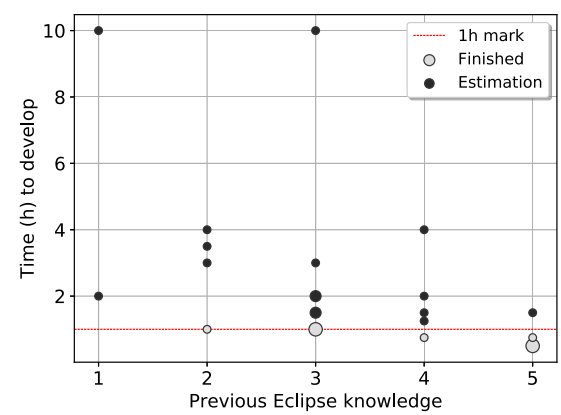

(d) Eclipse

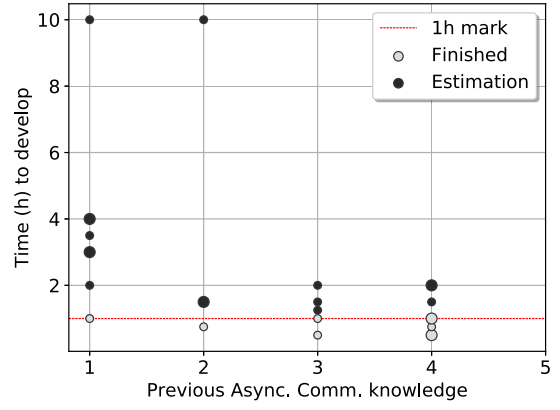

(b) Asynchronous Communications

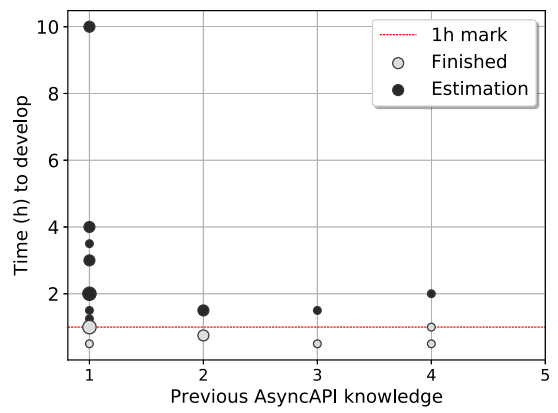

(e) AsyncAPI

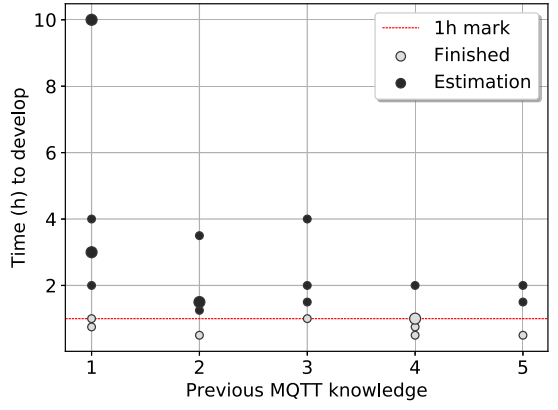

(c) $\mathrm{MQTT}$

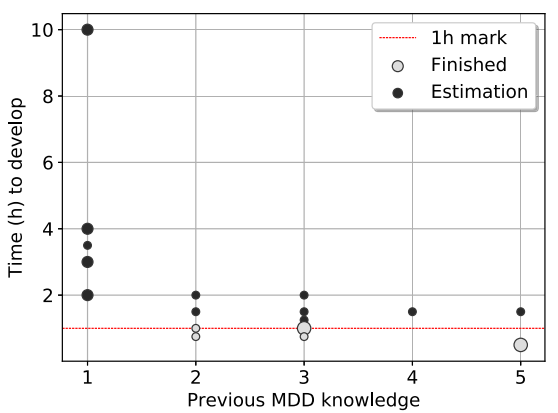

(f) $\mathrm{MDD}$

Fig. 9 Time to finish the exercise shows no direct correlation with previous knowledge on a Java, $\mathbf{b}$ asynchronous communication, $\mathbf{c}$ MQTT, $\mathbf{d}$ Eclipse, e AsyncAPI, and f MDD

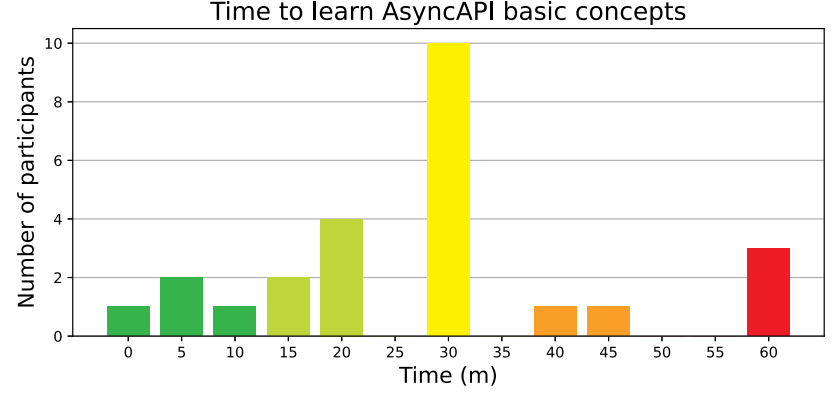

Fig. 10 Qualitative results

The chart depicted in the figure shows the time spent by the participants to read and get the basic knowledge about the AsyncAPI specification and AsyncAPI Toolkit to be able to start with the exercise. In average, the subjects only required $\approx \mathbf{2 8}$ minutes to get familiar with the specification and the tool to be ready to start with the proposed exercise.

Figure 10 shows other evaluated facets related to AsyncAPI Toolkit, as clarity of the documentation and the overall reliability among others. Those facets were rated in a 1 to 5 scale-from unsatisfactory to very satisfactoryrespectively. The obtained results are very positive.

Most of the subjects found the tool fairly intuitive (as we have just seen, the time to get familiar with the tool is quite small). The documentation completeness and organization, along with its clarity, have been positively rated. The subjects were also asked about the ease of installing and updating the application, with overall positive responses, standing out the easiness of installation.

Regarding the overall reliability and performance, few participants rated the AsyncAPI Toolkit low. Only around $4 \%$ of the participants found that it needs to improve its reliability and performance. Additionally, they were also asked about the development workflow around the tool. Almost all of them (92\%) answered that the workflow of the AsyncAPI Toolkit is the correct one, with the missing $8 \%$ equally divided between negative and "don't know" answers, $4 \%$ each.

In general, we observed that AsyncAPI Toolkit was highly rated on a global scoring and that most participants would recommend using it for implementing solutions that leverage asynchronous communications.

The open questions allowed us to answer RQ3 (weaknesses and strengths of the toolkit), and dig deeper in the overall perception of the AsyncAPI Toolkit and helped us to find aspects for improvement. In this regard, one of such questions was whether the subjects think the tool reduces the development and deployment time and how. The subjects unanimously answered that AsyncAPI Toolkit helps reducing development and deployment time. Also, given 


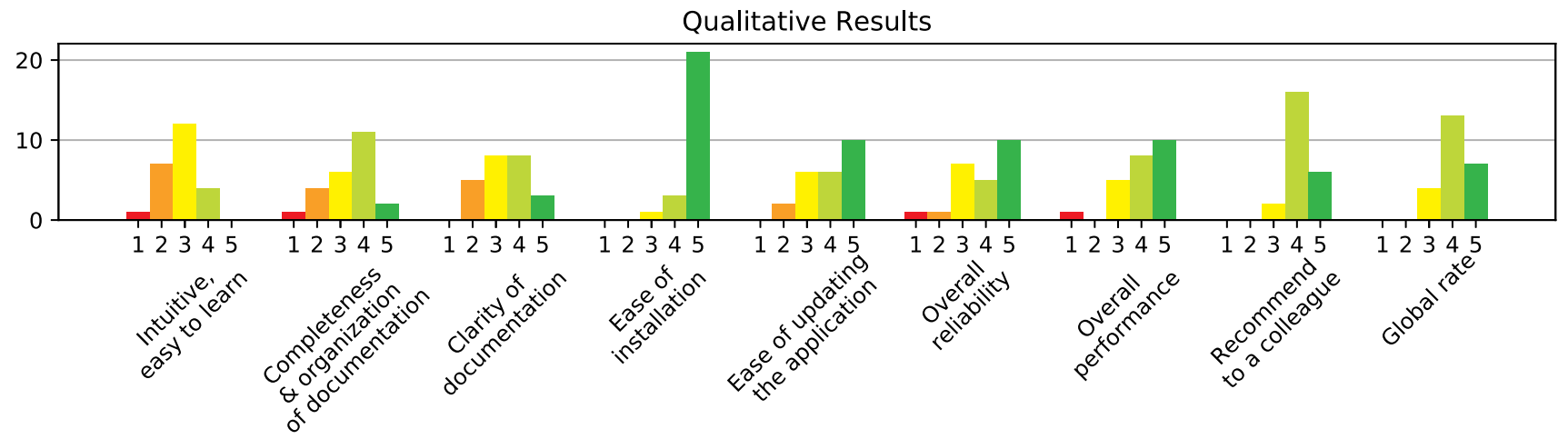

Fig. 11 Time spent learning the AsyncAPI basics

the overlapping between RQ1 (perceived benefits) and RQ3 (weaknesses and strengths), these open questions allowed us to confirm the results of the internal validation with the answers given by the external subjects. The following points were highlighted in the answers ${ }^{25}$ :

- Automatic code generation makes faster and less error prone the development process while saving time. "Everything concentrates in a single file and once it is filled and validated, the code is automatically generated".

- Model-based development simplifies the definition of complex tasks reducing time. "Not implementing the classes [of the DSL] saves time".

- Focus on design. Users can focus on designing the system and its API. "Developers waste less time on configuring and managing the API workflow, and they focus on designing the API".

- Abstraction. Users do not need to have big knowledge of the communication protocol, they only need to focus on defining the message content. "The user only needs to define the content of the messages to be transmitted since the tool abstracts the user from knowing the details of the communication protocol".

- Modifications. The tool facilitates modifications by the use of a single source of truth, as the classes are generated automatically, and only the AsyncAPI specification and the main application need to be changed. "Automatic code generation reduces development time and using models facilitates modification reducing time too".

- Reuse. What is generated can be reused in the application. The API definition can be easily reused and modified in similar projects too. "You can reuse the generated API directly in your application".

Another open question was what features would the participants add to AsyncAPI Toolkit. The responses included

\footnotetext{
$\overline{25}$ Quoted phrases have been directly extracted from subjects' declarations.
}

pointers to possible improvements, e.g., adding a way to edit the model graphically or using a tree view, improving the AsyncAPI textual editor itself, improving error detection and providing feedback while editing, and adding support for more programming languages. Some of these points are further expanded in Sect. 8.

Second Phase-This section discusses our findings from the interviews carried out after the second exercise, where we asked the subjects to solve the proposed exercise by using an annotated Ecore data model, and answers RQ4 (benefits of the graphical syntax).

All subjects agreed that using a graphical editor to bootstrap a working specification was a more visual and helpful way to develop the AsyncAPI definition. However, some subjects highlighted that this way of modeling, i.e., using Ecore annotations, could appeal more to people with metamodeling experience.

One of the participants pointed that the learning curve was lower using the graphical editor and the specification could be defined quicker in addition to the benefit of using the same environment that they already use in other modeling tasks.

The model-driven approach provided by the tool was rated as useful and practical. Some subjects noted that the tool integrated seamlessly in Eclipse and that it helped any modeler to create AsyncAPI definitions and the corresponding scaffolding easily.

The participants mentioned several advantages related to the graphical editor. They are summarized in the following points:

- Less error prone. The graphical view helps avoiding syntax and typing errors.

- Higher abstraction. Users have a higher abstraction model that may facilitate understanding and implementing complex systems.

- Better user experience. The majority agreed in that it was a better user experience. However, one participant with lesser modeling expertise preferred the textual editor. 
Meanwhile, the disadvantages found by those participants were:

- Burdensome editing procedure. "Point-and-click" repetitive procedure when modeling and editing the AsyncAPI specification may become cumbersome.

- Requires knowledge of metamodel annotations. While for people with expertise on metamodeling the feature was an advantage, participants without knowledge of metamodel annotations did not find it so easy.

Overall, when subjects were asked about comparing both ways of specifying the system, they all unanimously agreed that using a graphical editor to create an annotated data model made it easier to understand the problem domain at a glance. However, since the AsyncAPI specific information is embedded as annotations in the model, the user has to make an additional effort to understand how to map such information into the model elements. Also, it is not always clear how these annotations impact in the generated code, and this feature may require more knowledge and control on the modeled system.

As additional comments, the participants missed a kind of guided editor that facilitates the specification. For example, each time a channel is defined by the user, associated messages and schemas are automatically created even if no annotations for them are defined, and this is not obvious at first sight. Few participants brought up that documentation should be extended with other examples to facilitate the acquisition of knowledge. And a participant also pointed out the possibility to define an Unified Modeling Language (UML) profile for AsyncAPI metadata rather than annotations. This would ease the annotation phase using predefined stereotypes.

Regardless of this, everybody agreed the graphical editor clearly added value to the tool. As a participant noted, "it is easier and faster to model using the tool than starting from scratch".

\section{Related Work}

In this section, we compare our proposal with other works around API specifications, IIoT languages and code generators, and model-based approaches both for data and communication.

As we will see, most of previous work that target modeling data and communication aspects focus on synchronous architectures. The support for message-driven ones, like the one we propose, is much more limited.

We first start by covering core modeling proposals for IIoT (Sect. 7.1). Then, we move to works focusing on model-based IIoT proposals relying on synchronous communication- mostly REST-based ones-in Sect. 7.2. Finally, model-based IIoT proposals on top of asynchronous architectures are covered in Sect. 7.3.

\subsection{Domain-Specific Languages for IloT}

As we propose, MDE has already been used to accelerate the development process of industrial systems in the Industry 4.0 context. Among other works, the benefits of MDE for IIoT have been previously analyzed by Capilla et al. [8] and Young et al. [57], including some guidelines when modeling such systems to maximize their effectiveness [12].

Several works have explored the use of MDE in IoT at a global level. This is the case of Ciccozzi and Spalazzese [10], where the authors present the MDE4IoT framework supporting the modelling and self-adaptation of Emergent Configurations of connected systems. Clark and Barn [11] is a UML extension to define components that could be used in both service-oriented and event-driven architectures. AutoIoT [36] provides a generic metamodel and tool that designers can use to model their IoT scenarios either graphically (Web GUI) or textually (manually writing a JSON file). Models created with AutoIoT could then be the input of a model-to-text transformation to generate different implementations of the IoT application. ThingML [34] combines well-proven software-modeling constructs aligned with UML (statecharts and components) and an imperative platform-independent action language to specify IoT applications. Due to its popularity, other languages include a transformation to ThingML [46]. However, modeling of communication in ThingML is rather minimal. Similarly, Artikov et al. [4] defend the need to combine several modeling languages, each targeting a specific IoT concern, for a more consistent overall definition of the IoT system.

Indeed, these frameworks can be regarded as complementary to our own as they are more targeted to describing and implementing the IoT components themselves while we focus on describing the communication and interoperability among them. Next subsections cover proposals comprising as well the communication aspects, grouping them based on whether they mainly assume a synchronous or asynchronous communication.

\subsection{Model-based approaches for synchronous communications}

Several DSLs to model specific parts of IoT synchronous communication systems have been explored. One of such approaches is the one presented by Sneps-Sneppe and Namiot [48], where the authors present an extension of Java Server Pages to generate a web-based DSL to use in IoT applications. The proposed DSL enables IoT communications 
between the devices that support the process and the sensors. Negash et al. [35] also propose a DSL that is specifically designed for IoT, namely DoS-IL. However, they go further and also created an interpreter for the DOM, allowing the browser to be manipulated through scripts that interact with the DOM. CREST, presented by Klikovits et al. [29], is another DSL that aims to model CPSs of small scale that has synchronous evolution and reactive behavior. Riedel et al. [44] present a tool that generates C, C\# and Java code relying on SOAP Web Services (WS-SOAP) as the communication protocol. They propose the use of the Essential Meta-Object Facility (EMOF) [40] for data metamodels and EMF to generate the messages between IoT subsystems. Another approach is TRILATERAL [22,25], a tool that uses MDE with IoT communication protocols to generate artifacts for industrial CPSs. This tool allows using a visual editor to input a model based on the IEC 61850 standard for electrical substations and the tool automatically generates the $\mathrm{C}++$ code that enables the devices to communicate, so far, using HTTP-REST, CoAP, or WS-SOAP (all of them synchronous).

A more recent initiative is the Web of Things (WoT) [55], which focuses on facilitating the interoperability among devices thanks to a shared definition of a common data model. A couple of modeling proposals around WoT are Delicato et al. [13] and Iglesias-Urkia et al. [23,24]. While WoT could support some level of asynchronous communication around its event concept, it mainly targets synchronous architectures.

Being RESTful architectures the most significant example of synchronous architectures, several approaches are implemented following this architectural style. For instance, Thramboulidis and Christoulakis [54] integrate CPSs and IoT with a framework named UML4IoT that allows automating the process of generating CPSs. To do that, the CPSs are modeled using SysML and implemented using an objectoriented API that is later transformed to a RESTful API, using LWM2M for the communication. Ivanchikj and Pautasso [26] present RESTalk, a DSL for modeling and visualizing RESTful conversations, i.e., a model of all possible sequences of HTTP message exchanges between client and servers. As before, RESTalk is based on the model of the Ope$n A P I$ Specification and provides a visual and textual DSL.

OpenAPI (formerly Swagger) [47] is a predominant effort to allow describing RESTful APIs [50]. The OpenAPI initiative offers several tools to assist developers-e.g., editors, document generators, code generators, etc.- - proposed either by the consortium itself or by a growing ecosystem of thirdparty providers-e.g., APIs.guru [3]. We also start to see model-based tools for OpenAPI [16,17]. There are also some works that aim to infer API definitions from examples of calls or data points $[7,15]$ and may offer an intermediate model to be annotated before the generation of the (OpenAPI) API description file.
OPC-UA [32] is a SOA-based popular alternative to RESTful architectures in the industrial automation domain. A core element in any OPC-UA definition is the information model aimed at representing the static and dynamic behavior of manufacturing systems. Several approaches, mostly based on UML extensions, have been proposed to facilitate the specification of such information models $[30,42,43]$.

Many aspects of all these proposals have inspired key elements of the asynchronous approaches described next. The best example is AsyncAPI itself, which is heavily inspired by OpenAPI. However, due to the fundamental differences in the communication paradigm, previous approaches and tools cannot be directly reused in an AsyncAPI context as they are missing many of the key constructs to describe AsyncAPIor more generally, asynchronous-definitions like channel, subscription, server or message.

\subsection{Model-based approaches for message-driven architectures}

Beyond AsyncAPI, asynchronous communication can also be defined with Data Distribution Service (DDS) [37], which is a data-centric publish/subscribe (DCPS) model for distributed application communication. While both DDS and AsyncAPI provide a specific language for data definition - the so-called Interface Definition Language (IDL) [39] in the case of the former, and JSON Schema in the case of the latter-they differ in different aspects: (i) DDS is oriented to the data itself and not the messages [2]; (ii) DDS is technology agnostic, and as a consequence, does not consider architectural elements such as the different protocols, servers and listening ports- to name a few-involved; and (iii) DDS has a more mature ecosystem.

However, although DDS is technology agnostic, its implementations are more niche-focused ${ }^{26}:$ most of the solutions focus on supporting $\mathrm{C}$ and $\mathrm{C}++$ and support to other languages are provided via bridges to execute native code in compiled libraries. For instance, OpenDDS ${ }^{27}$ is an implementation of the DDS protocol that allows to define a data model with an IDL, and the code generator creates the data structure and a wrapper to access it. It creates $\mathrm{C}++$ code that can be accessed using the Java Native Interface (JNI). Another commercial tool is IBM's Rhapsody ${ }^{28}$, which allows to create DDS services in $\mathrm{C}++$ or Ada. The final available tool worth mentioning is RTI Code Generator ${ }^{29}$, which is a

\footnotetext{
26 https://www.dds-foundation.org/dds-resources/.

27 https://opendds.org/.

28 https://www.ibm.com/docs/en/rhapsody/8.2?topic=tutorialscreate-data-distribution-service-real-time-systems-application .

29 https://community.rti.com/static/documentation/connextdds/5.2.3/doc/manuals/connext_dds/code_generator/ RTI_CodeGenerator_UsersManual.pdf
} 
command line tool that uses an IDL or XML file as input and generates the code (Ada, C, C++, CLI, C\# or Java) for the data structure, and both publisher and subscriber examples. However, the main downsides of environments supporting DDS is that they are less integrated, with a lower level of abstraction, and no support for internal DSLs-as AsyncAPI Toolkit proposes with its fluent API - is provided.

CyprIoT [6] includes modeling constructs to describe the communication channels and hierarchy of topics and subtopics but does not address the structure of the internals of the exchanged messages.

Finally, and specifically focusing on AsyncAPI, while the ecosystem of tools around it keeps growing at a fast pace ${ }^{30}$, most of the tools still lack of some key features or are hard to integrate with external tools. For example, the Java AsyncAPI project $^{31}$ provides an implementation of the AsyncAPI specification in Java, similarly to what our AsyncAPI metamodel allows-Ecore metamodels are projected to Java code in EMF. However, although the Java AsyncAPI project allows dealing with AsyncAPI definitions in Java, it does not provide any code generation capabilities as the AsyncAPI Toolkit does.

Regarding code generation, the AsyncAPI Initiative now provides a generic template-based generator ${ }^{32}$ which can be used to generate code in any language. As of writing this manuscript, only the following languages are officially supported by the AsyncAPI generator: HTML and Markdown for documentation; and Node.js, Java (relying on Spring), Python, and TypeScript NATS for executable code. In all cases, only a subset of the protocols considered by Asyn$c A P I$ are supported (although MQTT is generally-but not always-supported), and in some cases, some optional properties of the AsyncAPI Specification are marked as required for the code generators to work ${ }^{33}$ thus breaking the compliance with the specification. In concrete the case of the Java code generator, the main difference with the AsyncAPI Toolkit is the lack of an internal DSL which helps developers to easily discover the data schema of the messages interchanged. Also, some importante features, such as parameters in topic names or server variables, are lacking or not fully supported. Finally, as opposed to our AsyncAPI Toolkit, which is implemented in Java for convenience purposes, the AsyncAPI generator is implemented in Node.js, which makes difficult to integrate it in a Java-based IDE. This is of special importance when the IDE also provides a plethora of MDE tools-as it is the case of Eclipse and EMF, which have become a de facto standard in the model-

\footnotetext{
$30 \mathrm{https} / / / \mathrm{www}$. asyncapi.com/docs/community/tooling.

31 https://github.com/Pakisan/java-asyncapi.

32 https://github.com/asyncapi/generator.

33 E.g.., the operationId property must be always set to use the Node.js generators
}

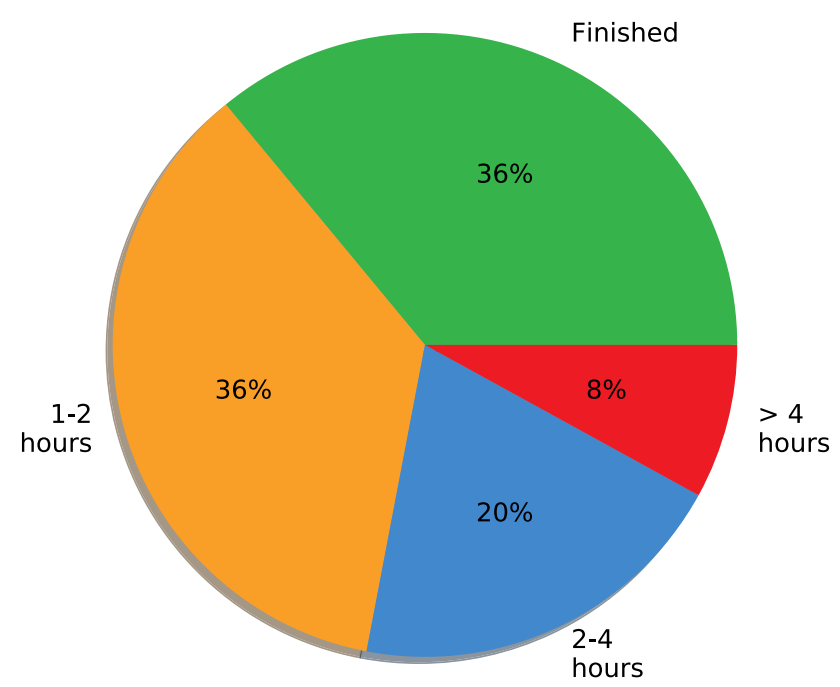

Fig. 12 Percentage of people finishing in $1 \mathrm{~h}, 2 \mathrm{~h}, 4 \mathrm{~h}$ or more

ing community - which could help to build more complete and mature model-based tools around AsyncAPI. Thus, ours is the only approach targeting the modeling of AsyncAPI definitions providing an internal DSL in the form of a fluent API based on data models, and providing an IDE to develop the AsyncAPI definitions with full code generation capabilities in a single solution.

\section{Conclusions and further work}

This article presents AsyncAPI Toolkit, a toolkit that allows specifying message-driven APIs using the AsyncAPI specification also providing automatic code generation. AsyncAPI Toolkit decreases the development time of architectures with asynchronous communication.

As described in the previous section, the investment in time to learn the basics of AsyncAPI and the AsyncAPI Tool$k i t$ is not too much. Even though few of the participants had previous knowledge of AsyncAPI, with a mean learning time of $\approx 28$ minutes, $72 \%$ of the participants finished the exercise within the given hour or estimate that they would need less than a total of two hours to finish it as it is shown in Fig. 12. And the $36 \%$ of the participants were able to finish it within the fixed hour with only that mean learning time spent for initial knowledge acquisition.

Investing a bit more time in learning the basics of Asyn$c A P I$ should lead to better results in fewer time and effort. But for those developers who do not know the AsyncAPI specification in depth and do not have the need to deepen on it, the AsyncAPI Toolkit has proven to be a tool able to rapidly provide an interface to their systems.

Not only developers can get benefit from the AsyncAPI Toolkit, but also modelers can. The extensions discussed in 
Sects. 4.2, 5.3 and 5.4 are addressed to these users, who may expect this kind of functionality in any model-driven solution as they are used to it. In fact, after the first phase of the evaluation, most of the participants familiar with MDD requested or asked about a higher-level graphical editor. As a consequence, the AsyncAPI Toolkit allows starting a new API definition in two different ways: either creating a data model using an Ecore editor which generates an initial AsyncAPI definition, or directly creating the AsyncAPI definition textually.

Considering all participants suggestions and our own experience, these are the main lines of future work in our roadmap:

1. Automatic test suite generation: Having a new test generation functionality will facilitate the validation of the communication system's robustness. The goal would be to automatically generate different types of communication messages (both valid and invalid ones) considering the usual vulnerabilities that make not to comply the single source of truth. This mechanism will help to anticipate to possible problems and assure that the system will not be down due to those common issues.

2. Cover other languages besides Java: This has been highly requested. Our next milestone will include additional code generation templates to be able to automatically generate code according to the project's development language target: Python, C, C++, etc.

3. User experience improvement: Better error detection and identification during the specification editing, quick fix features to correct them, enhance AsyncAPI specification file editing with a tree view editor and include the requested editor assistant or wizards for the development of the model will all be part of our planned improvements in this line of work. Although they do not represent a new functionality for the AsyncAPI Toolkit, it makes a difference in user satisfaction and we think it will also improve the development time as nowadays error detection and fixing are not so intuitive for the user.

Finally, we will also evaluate AsyncAPI Toolkit further in to different aspects. On the one hand, we plan to perform additional empirical validations that help us better understand the specific trade-offs of introducing our model-driven AsyncAPI infrastructure in new industrial projects, when we get a larger dataset for this analysis and explore the option to employ our toolkit in reverse engineering scenarios, e.g., to generate visualizations of existing AsyncAPI specifications with our graphical syntax. On the other hand, we also plan to compare AsyncAPI Toolkit against other tools that undoubtedly will be developed based on AsyncAPI, following a systematic review approach such as the one described in [9].
Acknowledgements This project has received funding from the Electronic Component Systems for European Leadership Joint Undertaking under grant agreement $\mathrm{N}^{\circ} 737494$, this Joint Undertaking receives support from the European Union's Horizon 2020 research and innovation program and from Sweden, France, Spain, Italy, Finland \& Czech Republic; from the European commission under the COMP4DRONES project under grant agreement $\mathrm{N}^{\circ} 826610$ with support from the ECSEL-JU 2018 program with national financing from France, Spain, Italy, Netherlands, Austria, Czech, Belgium and Latvia; and from the Spanish government under project Open Data for All (RETOS TIN2016-75944-R). We also want to acknowledge all participants for their collaboration in the experiment.

Funding Open Access funding provided thanks to the CRUE-CSIC agreement with Springer Nature.

Open Access This article is licensed under a Creative Commons Attribution 4.0 International License, which permits use, sharing, adaptation, distribution and reproduction in any medium or format, as long as you give appropriate credit to the original author(s) and the source, provide a link to the Creative Commons licence, and indicate if changes were made. The images or other third party material in this article are included in the article's Creative Commons licence, unless indicated otherwise in a credit line to the material. If material is not included in the article's Creative Commons licence and your intended use is not permitted by statutory regulation or exceeds the permitted use, you will need to obtain permission directly from the copyright holder. To view a copy of this licence, visit http://creativecomm ons.org/licenses/by/4.0/.

\section{References}

1. Al-Fuqaha, A., Guizani, M., Mohammadi, M., Aledhari, M., Ayyash, M.: Internet of things: a survey on enabling technologies, protocols, and applications. IEEE Commun. Surv. Tutor. 17(4), 2347-2376 (2015)

2. Alaerjan, A., Kim, D.K., Ming, H., Malik, K.: (2018) Using DDS based on unified data model to improve interoperability of smart grids. In: 2018 6th IEEE International Conference on Smart Energy Grid Engineering, SEGE 2018, pp 110-114, https://doi.org/10. 1109/SEGE.2018.8499513

3. APIs.guru API tooling for better developer experience. last accessed September (2020)https://apis.guru/ (2020)

4. Artikov, M., Meier, J., Winter, A. :Towards integrated iotlanguages. In: 2019 International Conference on Information Science and Communications Technologies (ICISCT), pp 1-5, (2019) https://doi.org/10.1109/ICISCT47635.2019.9011964

5. AsyncAPI Initiative AsyncAPI specification 2.0.0. URL: https:// www.asyncapi.com/docs/specifications/2.0.0/, last accessed May $2021(2020)$

6. Berrouyne, I., Adda, M., Mottu, J., Royer, J., Tisi, M.: Cypriot: framework for modelling and controlling network-based iot applications. In: Hung C, Papadopoulos GA (eds) Proceedings of the 34th ACM/SIGAPP Symposium on Applied Computing, SAC 2019, Limassol, Cyprus, April 8-12, 2019, ACM, pp 832-841, (2019)https://doi.org/10.1145/3297280.3297362

7. Cánovas-Izquierdo, J.L., Cabot, J.: Jsondiscoverer: visualizing the schema lurking behind JSON documents. Knowl. Based Syst. 103, 52-55 (2016). https://doi.org/10.1016/j.knosys.2016.03.020

8. Capilla, R., Bosch, J., Trinidad, P., Ruiz-Cortés, A., Hinchey, M.: An overview of dynamic software product line architectures and techniques: observations from research and industry. J. Syst. Softw. 91, 3-23 (2014) 
9. Challenger, M., Kardas, G., Tekinerdogan, B.: A systematic approach to evaluating domain-specific modeling language environments for multi-agent systems. Softw. Qual. J. 24(3), 755-795 (2016). https://doi.org/10.1007/s11219-015-9291-5

10. Ciccozzi, F., Spalazzese, R.: MDE4IoT: Supporting the internet of things with model-driven engineering. In: Badica, C., El Fallah, Seghrouchni A., Beynier, A., Camacho, D., Herpson, C., Hindriks, K., Novais, P. (eds.) Intelligent Distributed Computing X, pp. 6776. Springer International Publishing, Cham (2017)

11. Clark, T., Barn, B.S.: A common basis for modelling serviceoriented and event-driven architecture. In: Proceedings of the 5th India Software Engineering Conference, Association for Computing Machinery, New York, NY, USA, ISEC '12, p 23-32, (2012) https://doi.org/10.1145/2134254.2134258

12. Das, T.K., Dingel, J.: Model development guidelines for UML-RT: conventions, patterns and antipatterns. Softw. Syst. Model. 17(3), 717-752 (2018). https://doi.org/10.1007/s10270-016-0549-6

13. Delicato, F.C., Pires, P.F., Batista, T.: Middleware Solutions for the Internet of Things. Springer Publishing Company Incorporated, London (2013)

14. Díaz, O., Villoria, F.M.: Generating blogs out of product catalogues: An MDE approach. J. Syst. Softw. 83(10), 1970-1982 (2010). https://doi.org/10.1016/j.jss.2010.05.075

15. Ed-Douibi, H., Cánovas-Izquierdo, J.L., Cabot, J.: Example-driven web API specification discovery. In: Modelling Foundations and Applications-13th European Conference, ECMFA@STAF 2017, Marburg, Germany, July 19-20, 2017, Proceedings, pp 267-284, (2017)https://doi.org/10.1007/978-3-319-61482-3_16

16. Ed-Douibi, ,H., Cánovas-Izquierdo, J.L., Cabot, J.: Openapitouml: A tool to generate UML models from openapi definitions. In: Proceedings of Web Engineering-18th International Conference, ICWE 2018, Cáceres, Spain, June 5-8, 2018, pp 487-491, (2018). https://doi.org/10.1007/978-3-319-91662-0_41

17. Ed-Douibi, H., Izquierdo, J.L.C., Cabot, J.: Automatic generation of test cases for REST apis: A specification-based approach. In: 22nd IEEE International Enterprise Distributed Object Computing Conference, EDOC 2018, Stockholm, Sweden, October 16-19, 2018, pp 181-190,(2018) https://doi.org/10.1109/EDOC. 2018.00031

18. Evans, D.: The internet of things: How the next evolution of the internet is changing everything. CISCO white paper 1(2011), 1-11 (2011)

19. Fielding, R.T.: REST: architectural styles and the design of network-based software architectures. Doctoral dissertation, University of California, Irvine (2000)

20. Fowler, M.: Domain Specific Languages, 1st edn. Addison-Wesley Professional, Boston (2010)

21. Gómez, A., Iglesias-Urkia, M., Urbieta, A., Cabot, J.: A modelbased approach for developing event-driven architectures with asyncapi. In: Proceedings of the 23rd ACM/IEEE International Conference on Model Driven Engineering Languages and Systems, Association for Computing Machinery, New York, NY, USA, MODELS '20, pp. 121-131, https://doi.org/10.1145/3365438. 3410948 (2020)

22. Iglesias, A., Iglesias-Urkia, M., López-Davalillo, B., Charramendieta, S., Urbieta, A.: Trilateral: Software product line based multidomain iot artifact generation for industrial cps. In: Proceedings of the 7th International Conference on Model-Driven Engineering and Software Development - Volume 1: MODELSWARD, INSTICC, SciTePress, pp 64-73, https://doi.org/10. 5220/0007343500640073 (2019)

23. Iglesias-Urkia, M., Gómez, A., Casado-Mansilla, D., Urbieta, A.: Enabling easy web of things compatible device generation using a model-driven engineering approach. In: ACM International Conference Proceeding Series, (2019). https://doi.org/10. $1145 / 3365871.3365898$
24. Iglesias-Urkia, M., Gómez, A., Casado-Mansilla, D., Urbieta, A.: Automatic generation of web of things servients using thing descriptions. Pers. Ubiquit. Comput. (2020). https://doi.org/10. 1007/s00779-020-01413-3

25. Iglesias-Urkia, M., Iglesias, A., López-Davalillo, B., Charramendieta, S., Casado-Mansilla, D., Sagardui, G., Urbieta, A.: Trilateral: A model-based approach for industrial cps-monitoring and control. In: Hammoudi, S., Pires, L.F., Selić, B. (eds.) Model-Driven Engineering and Software Development, pp. 376-398. Springer International Publishing, Cham (2020)

26. Ivanchikj, A., Pautasso, C.: Modeling microservice conversations with restalk. In: Bucchiarone A, Dragoni N, Dustdar S, Lago P, Mazzara M, Rivera V, Sadovykh A (eds) Microservices: Science and Engineering, Springer International Publishing, Cham, pp 129146, (2020). https://doi.org/10.1007/978-3-030-31646-4_6

27. Jazdi, N.: Cyber physical systems in the context of industry 4.0. In: 2014 IEEE International Conference on Automation, Quality and Testing, Robotics, pp 1-4, (2014). https://doi.org/10.1109/AQTR. 2014.6857843

28. Kagermann, H., Helbig, J., Hellinger, A., Wahlster, W.: Recommendations for implementing the strategic initiative INDUSTRIE 4.0: Securing the future of German manufacturing industry; final report of the Industrie 4.0 Working Group. Forschungsunion (2013)

29. Klikovits, S., Linard, A., Buchs, D.: Crest - a dsl for reactive cyberphysical systems. In: Khendek F, Gotzhein R (eds) System Analysis and Modeling. Languages, Methods, and Tools for Systems Engineering, Springer International Publishing, Cham, pp 29-45 (2018)

30. Lee, B., Kim, D.K., Yang, H., Oh, S.: Model transformation between OPC UA and UML. Comput. Stand. Interface. 50, 236250 (2017). https://doi.org/10.1016/j.csi.2016.09.004

31. Leitão, P., Colombo, A.W., Karnouskos, S.: Industrial automation based on cyber-physical systems technologies: Prototype implementations and challenges. Comput. Ind. 81, 11-25 (2016)

32. Leitner, S.H., Mahnke, W.: Opc ua-service-oriented architecture for industrial applications. ABB Corpor. Res. Center 48, 61-66 (2006)

33. Michelson, B.M.: Event-driven architecture overview. Patricia Seybold Group 2(12), 10-1571 (2006)

34. Morin, B., Harrand, N., Fleurey, F.: Model-based software engineering to tame the iot jungle. IEEE Softw. 34(1), 30-36 (2017). https://doi.org/10.1109/MS.2017.11

35. Negash, B., Westerlund, T., Rahmani, A.M., Liljeberg, P., Tenhunen, H.: Dos-il: A domain specific internet of things language for resource constrained devices. In: Procedia Computer Science, Elsevier B.V., vol 109, pp. 416-423, (2017). https://doi.org/10.1016/j. procs.2017.05.411

36. Nepomuceno, T., Carneiro, T., Maia, P.H., Adnan, M., Nepomuceno, T., Martin, A.: (2020) Autoiot: A framework based on user-driven mde for generating iot applications. In: Proceedings of the 35th Annual ACM Symposium on Applied Computing, ACM, SAC '20, p 719-728, 10.1145/3341105.3373873

37. OMG (2015) Data Distribution Service (DDS), Ver. 1.4. https:// www.omg.org/spec/DDS/1.4/

38. OMG (2017) OMG Unified Modeling Language (OMG UML), Ver. 2.5.1. https://www.omg.org/spec/UML/2.5.1/

39. OMG (2018) Interface Definition Language (IDL), Ver. 4.2. https:// www.omg.org/spec/IDL/4.2/

40. OMG (2019) Meta Object Facility (MOF), Ver. 2.5.1. http://www. omg.org/spec/MOF/2.5.1/

41. OpenAPI Initiative (2020) OpenAPI Specification. URL: https:// github.com/OAI/OpenAPI-Specification, last accessed May 2021

42. Pauker, F., Frühwirth, T., Kittl, B., Kastner, W.: A Systematic Approach to OPC UA Information Model Design. Procedia CIRP 57, 321-326 (2016). https://doi.org/10.1016/j.procir.2016.11.056, factories of the Future in the digital environment - Proceedings of the 49th CIRP Conference on Manufacturing Systems 
43. Pauker, F., Wolny, S., Fallah, S.M., Wimmer, M.: UML2OPC-UA Transforming UML Class Diagrams to OPC UA Information Models. Procedia CIRP 67, 128-133 (2018). https://doi.org/10.1016/j. procir.2017.12.188, 11th CIRP Conference on Intelligent Computation in Manufacturing Engineering, 19-21 July 2017, Gulf of Naples, Italy

44. Riedel, T., Fantana, N., Genaid, A., Yordanov, D., Schmidtke, H.R., Beigl, M.: Using web service gateways and code generation for sustainable iot system development. In: 2010 Internet of Things (IOT), Tokyo, Japan, pp 1-8, 10.1109/IOT.2010.5678449 (2010)

45. Schwab K (2017) The Fourth Industrial Revolution. Crown Publishing Group

46. Sharaf, M., Abusair, M., Eleiwi, R., Shana'a, Y., Saleh, I., Muccini, H.: Modeling and code generation framework for iot. In: i Casas PF, Sancho M, Sherratt E (eds) System Analysis and Modeling. Languages, Methods, and Tools for Industry 4.0 - 11th International Conference, SAM 2019, Munich, Germany, September 16-17, 2019, Proceedings, Springer, Lecture Notes in Computer Science, vol 11753, pp 99-115, https://doi.org/10.1007/978-3-030-306908_6 (2019)

47. SmartBear Software (2020) What Is OpenAPI? https://swagger.io/ docs/specification/about/

48. Sneps-Sneppe, M., Namiot, D.:On web-based domain-specific language for internet of things. In: International Congress on Ultra Modern Telecommunications and Control Systems and Workshops, IEEE Computer Society, vol 2016-January, pp 287-292, https://doi.org/10.1109/ICUMT.2015.7382444 (2016)

49. Steinberg, D., Budinsky, F., Paternostro, M., Merks, E.: EMF: Eclipse Modeling Framework 2.0, 2nd edn. Addison-Wesley Professional, ISBN: 0321331885 (2009)

50. Tashtoush, Y., AlRashdan, M.N., Salameh, O., Alsmirat, M.: Swagger-based jquery ajax validation. In: 2019 IEEE 9th Annual Computing and Communication Workshop and Conference (CCWC), pp 0069-0072, https://doi.org/10.1109/CCWC. 2019.8666542 (2019)

51. The Eclipse Foundation (2020) Eclipse Modeling Project Eclipse Modeling Framework - Home. http://www.eclipse.org/ emf/, Accessed May 2021

52. The Eclipse Foundation (2020) Xtend - Modernized Java. http:// www.eclipse.org/xtend/. Accessed May 2021

53. The Eclipse Foundation (2020) Xtext - Language Engineering Made Easy! http://www.eclipse.org/Xtext/. Accessed May 2021

54. Thramboulidis, K., Christoulakis, F.: UML4IoT - A UML-based approach to exploit IoT in cyber-physical manufacturing systems. Comput. Ind. 82, 259-272 (2016). https://doi.org/10.1016/j. compind.2016.05.010

55. W3C (2019) Web of Things at W3C. https://www.w3.org/WoT/

56. Wright, H.K., Kim, M., Perry, D.E.: Validity concerns in software engineering research. In: Proceedings of the FSE/SDP Workshop on Future of Software Engineering Research, Association for Computing Machinery, New York, FoSER '10, p 411-414, (2010). https://doi.org/10.1145/1882362.1882446

57. Young, B., Cheatwood, J., Peterson, T., Flores, R., Clements, P.C.: Product line engineering meets model based engineering in the defense and automotive industries. In: Proceedings of the 21 st International Systems and Software Product Line Conference, SPLC 2017, Volume A, pp 175-179 (2017)

Publisher's Note Springer Nature remains neutral with regard to jurisdictional claims in published maps and institutional affiliations.

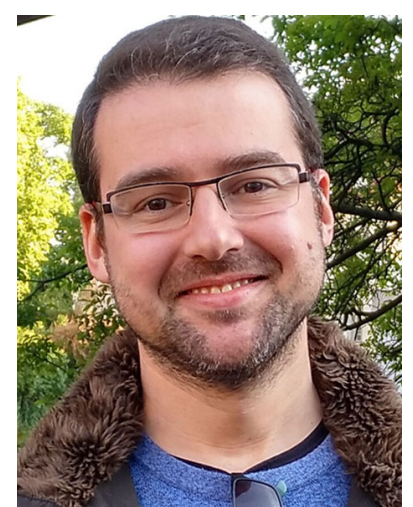

Abel Gómez is a senior researcher of the Internet Interdisciplinary Institute, a research center of the Universitat Oberta de Catalunya, Spain. Previously, he has hold different positions at the Universidad de Zaragoza, the École des Mines de Nantes \& Inria, and the Universitat Politécnica de València; being this latter institution where he obtained his $\mathrm{PhD}$ degree in Computer Science. His research interests fall in the broad field of Model-Driven Engineering (MDE), and his research lines have evolved in two complementary directions: on the one hand, the development of core technologies to support MDE activities; and on the other hand, the application of MDE techniques to solve Software Engineering problems. More information is available at https://abel. gomez.llana.me.

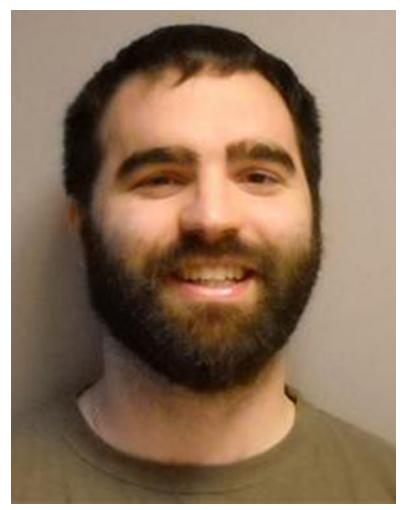

Markel Iglesias-Urkia is a researcher at Ikerlan on the IoT and Digital Platforms team since 2019, after completing his $\mathrm{PhD}$ on Engineering for the Information Society and Sustainable Development from the University of Deusto in the same team. Previously, he received his $\mathrm{BSc}$ on Computer Systems Engineering, BSc Computer Science \& Engineering and MSc on Embedded System Engineering from the University of the Basque Country. His research interests include Internet of Things (IoT), CoAP, lightweight protocols, Edge Computing among others. $\mathrm{He}$ is author or co-author of several peer-reviewed scientific publications in the field of the Internet of Things and Software Engineering.

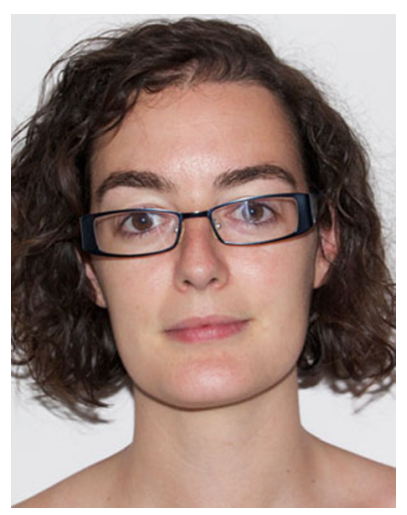

Lorea Belategi joined IKERLAN as a researcher in Dependable Software Team. After completing her $\mathrm{PhD}$ at Mondragon Unibertsitatea, she worked in diverse sectors (petrochemical, energy, health, etc.). After several years she became responsible for software quality at Kiro Grifols where she coordinated and carried out the tasks of verification and validation of medical devices software to be certified according to FDA (Food and Drug Administration). Later, she moved back to research, at IKERLAN. Her research interests include software V\&V, Edge Computing, Model-Driven Engineering (MDE), embedded systems. She is author or co-author of several peer-reviewed scientific publications in the field of model based software V\&V. 


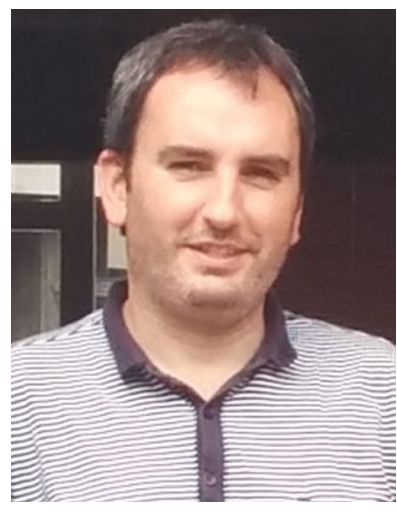

control systems.
Xabier Mendialdua is a researcher of the Dependable Software Team at Ikerlan S. Coop. Since 1994 he has been involved in multiple projects in several domains as automated warehouse management systems, monitoring systems, wind power and railway. His main activity is focused in the design and development of dependable software for embedded control systems and his research activities have been involved on applying model-driven and product-line approaches for the engineering of

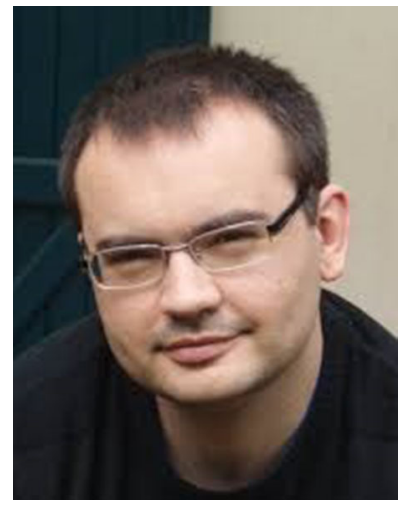

Jordi Cabot received the B.Sc. and $\mathrm{Ph} . \mathrm{D}$. degrees in computer science from the Technical University of Catalonia. He was a Leader of an INRIA and LINA Research Group at École des Mines de Nantes, France, a Post-Doctoral Fellow with the University of Toronto, a Senior Lecturer with the Open University of Catalonia, and a Visiting Scholar with the Politecnico di Milano. He is currently an ICREA Research Professor at Internet Interdisciplinary Institute. His research interests include software and systems modeling, formal verification and the role AI can play in software development (and vice versa). He has published over 150 peer-reviewed conference and journal papers on these topics. Apart from his scientific publications, he writes and blogs about all these topics in several sites. He is a member of the IEEE and the ACM. 